\title{
Selective Tuning for Contrast in Macaque Area V4
}

\author{
Ilaria Sani, ${ }^{1}$ Elisa Santandrea, ${ }^{1}$ Ashkan Golzar, ${ }^{2}$ Maria Concetta Morrone, ${ }^{3,4}$ and Leonardo Chelazzi ${ }^{1,5}$ \\ ${ }^{1}$ Department of Neurological and Movement Sciences, University of Verona, I-37129 Verona, Italy, ${ }^{2}$ Department of Physiology, McGill University, \\ Montreal, Quebec, H3A 0G4, Canada, ${ }^{3}$ Department of Translational Research on New Technologies in Medicine and Surgery, University of Pisa, I-56126 \\ Pisa, Italy, ${ }^{4}$ Stella Maris Foundation IRCCS, I-56128 Pisa, Italy, and ${ }^{5}$ National Institute of Neuroscience, I-37134 Verona, Italy
}

Visually responsive neurons typically exhibit a monotonic-saturating increase of firing with luminance contrast of the stimulus and are able to adapt to the current spatiotemporal context by shifting their selectivity, therefore being perfectly suited for optimal contrast encoding and discrimination. Here we report the first evidence of the existence of neurons showing selective tuning for contrast in area V4d of the behaving macaque (Macaca mulatta), i.e., narrow bandpass filter neurons with peak activity encompassing the whole range of visible contrasts and pronounced attenuation at contrasts higher than the peak. Crucially, we found that contrast tuning emerges after a considerable delay from stimulus onset, likely reflecting the contribution of inhibitory mechanisms. Selective tuning for luminance contrast might support multiple functions, including contrast identification and the attentive selection of low contrast stimuli.

\section{Introduction}

Along the visual hierarchy, neuronal responses to increasing levels of luminance contrast are well captured by sigmoid-like functions, such as the Naka-Rushton function (Albrecht and Hamilton, 1982), characterized by a monotonic increase in neuronal firing with stimulus contrast, typically followed by a plateau. Such contrast response functions (CRFs) are highly adaptable to the spatial and temporal context in which they are measured, and neurons shift their selectivity to optimally detect small variations in contrast with respect to the local mean (Enroth-Cugell and Shapley, 1973; Maffei et al., 1973; Movshon and Lennie, 1979; Sclar et al., 1989; Bonds, 1991; Sharpee et al., 2006). Monotonic CRFs and their adaptability are highly advantageous for optimal contrast encoding and discrimination.

In recent years, the interplay between spatial selective attention and stimulus contrast has been thoroughly investigated, mainly in area V4 (and MT), and multiple models have been proposed to describe the effects of attention on CRFs, including contrast gain (Reynolds et al., 2000), multiplicative rescaling (Williford and Maunsell, 2006), and additive models (Thiele et al., 2009). Although still awaiting systematic empirical confirmation, a computational model has attempted to reconcile such divergent results (the normalization model of attention; Lee and Maunsell, 2009; Reynolds and Heeger, 2009). With the ultimate

Received Aug. 12, 2013; revised Sept. 29, 2013; accepted 0ct. 15, 2013.

Author contributions: L.C. designed research;I.S., E.S., and A.G. performed research; I.S. and A.G. analyzed data; I.S., E.S., M.C.M., and L.C. wrote the paper.

This work was supported by funding from the Italian Government (Ministero dell'Istruzione, dell'Università, e della Ricerca; L.C.), European Research Council grant-Space, Time and Number in the Brain (M.C.M.), and International Brain Research Organization Studentship 2009 (A.G.). We thank Bjørg E. Kilavik for her contribution to initial developments of this work, Davide Zoccolan for helpful suggestions on the issue of cell classification, and Erik Cook for insightful comments on a previous version of this manuscript. We also thank Marco Veronese, Gianni Finizia, and, especially, Daniele Molinari for precious technical assistance.

The authors declare no competing financial interests.

Correspondence should be addressed to Leonardo Chelazzi, Department of Neurological and Movement Sciences, Section of Physiology and Psychology, University of Verona, Strada Le Grazie 8, I-37134 Verona, Italy. E-mail: leonardo.chelazzi@univr.it.

DOI:10.1523/JNEUROSCI.3465-13.2013

Copyright $\odot 2013$ the authors $\quad 0270-6474 / 13 / 3318583-14 \$ 15.00 / 0$ goal of advancing current understanding of the influence of spatially directed attention on neuronal responses to contrast, we maintain that fine characterization of CRFs and their temporal dynamics in area V4, as accomplished here, is fundamental for at least two reasons. First, the effects of attention on neuronal responses in area V4 (and other primate cortical visual areas) have been shown to vary as a function of time (McAdams and Maunsell, 1999; Reynolds et al., 2000); therefore, a full understanding of attentional influences on CRFs is likely to benefit greatly from an adequate characterization of the temporal dynamics of contrast encoding in this area. Second, studies addressing the impact of attention on CRFs have rather neglected any potential heterogeneity in contrast coding, likely including monotonically increasing, saturating, and supersaturating CRFs (but see Williford and Maunsell, 2006, for a preliminary effort in this direction).

The phenomenon of supersaturation (Maffei and Fiorentini, 1973), corresponding to an attenuation of neuronal responses in the high contrast range exceeding the saturation level, has mainly been described in V1 cells of the cat and monkey (Albrecht and Hamilton, 1982; Li and Creutzfeldt, 1984; Ledgeway et al., 2005; Peirce, 2007), whereas no clear assessment or quantification is available for V4 neurons. Although previously regarded just as an extreme manifestation of saturation, supersaturation has gained increasing interest in the recent past since a few studies have hypothesized a specific functional role for the phenomenon (Peirce, 2007, 2013; May and Zhaoping, 2011,2013), suggesting an improved capability of the visual system to encode contrast by relying on signals from neurons displaying monotonic and non-monotonic behavior (May and Zhaoping, 2011).

Here we report the results from experiments in which we were able to describe selective CRFs - with peak responses across the neuronal population encompassing the whole range of visible contrasts - by recording the spiking activity of macaque area V4 neurons in response to simple, aperiodic static stimuli (oriented achromatic bars) presented at varying levels of contrast. 


\section{Materials and Methods}

Experiments were performed on two head-fixed, non-anesthetized, male adult macaque monkeys (Macaca mulatta) weighing 10 and $8 \mathrm{~kg}$, respectively (monkey $\mathrm{F}$ and monkey $\mathrm{T}$ ). Use of the macaque monkeys and the experimental protocol were approved by the University of Verona Committee for Animal Research and by the Department for the Veterinary Public Health, Nutrition, and Food Security of the Italian Ministry of Health (Decreto Legislativo 116/1992, articolo 8/9; Decreto Ministeriale 19/2007c, 13/02/2007; and 200/2009c, 11/11/2009). The monkeys were housed and handled in strict accordance with the recommendations of the Weatherall Report about good animal practice, and their wellbeing and health conditions were constantly monitored by the institutional veterinary doctor.

Recordings were obtained while the animals were engaged in an orientation discrimination task; the contrast of the stimuli was task irrelevant, although it contributed significantly to the discriminability of target stimuli. This approach ensured that visual responses to contrast were uninfluenced by modulations related to the direct behavioral relevance of the property under study. In addition, to exclude any potential influence of variable attentional load for stimuli displayed at different contrast levels, we focused our study on conditions in which attention was allocated outside the receptive field (RF) and compared results with data collected in a control experiment in which the animals were engaged in a passive fixation task (see Results).

The use of spatial broadband stimuli (achromatic bars), which appear to be the most effective stimuli to characterize neurons in V1 output layers (Yeh et al., 2009), with abrupt onset and sustained in time, enabled us to explore the temporal dynamics of contrast encoding by the recorded neurons, and this was done for three principal reasons: (1) because we anticipated that a fine analysis of temporal dynamics might provide important hints as to the underlying circuit-level mechanisms; (2) because previous investigations have reported divergent results as to whether CRFs of cortical visual neurons are significantly modified during the temporal unfolding of stimulus-driven responses (Albrecht et al., 2002; Hu et al., 2011) and we wanted to provide evidence bearing on this issue; and (3) as stated above, an adequate characterization of the temporal dynamics of contrast encoding in area V4 might be critical for revisiting the analysis of attentional influences on CRFs, which are known to vary as a function of time (McAdams and Maunsell, 1999; Reynolds et al., 2000).

Surgical procedures and recording methods. Surgical procedures and recording methods have been described in detail previously (Mirabella et al., 2007). With respect to the recording of neuronal firing, a particular effort was made to exclude any multiunit contribution to the recorded activity, because this study aimed at finely describing and quantifying properties of individual neurons in macaque area V4. Spikes were discriminated using an online spike-sorting system [part of the data were acquired by using SPS-8701 (Signal Processing Systems) and part by using the Multichannel Acquisition Processor system (Plexon)] and acquired for offline analysis at $1 \mathrm{kHz}$ on a personal computer. In most cases, two (up to four) neurons could be recorded simultaneously and accurately differentiated on the basis of the size and shape of the spike waveform. Additional selection criteria were applied offline according to the stability of neuronal responses along the whole experimental session.

$R F$ mapping. Before the behavioral experiment was conducted, each well isolated cell was carefully characterized to determine its preference for orientation and spatial frequency (and sometimes for other stimulus properties, such as color) as well as its response to contrast while the monkey fixated centrally. The RF size was determined using the minimum response field method (Barlow et al., 1967). Bar stimuli of a fixed size $\left(2.2^{\circ} \times 0.3^{\circ}\right)$ were used throughout to be well within the classical RF boundary of the recorded neurons, in accordance with data in the literature (Motter, 2009) and as confirmed for each neuron during the initial mapping procedure by using flashing bars at different contrast levels, including high contrasts. Note that the minimum response field method for mapping RFs is known to underestimate RF size by a factor of 2-3 compared with the extending patches method (Walker et al., 2000; Cavanaugh et al., 2002).
Behavioral paradigm and stimuli. We trained two adult macaque monkeys to discriminate the orientation of achromatic bar stimuli in return for juice reward. Each trial began with the presentation of a fixation point (black square, $0.3^{\circ} \times 0.3^{\circ}$ of visual angle; Fig. $1 \mathrm{~A}$ ) on a dark background $\left(2.49 \mathrm{~cd} / \mathrm{m}^{2}\right)$. After the animal acquired fixation, a pentagon cue $\left(1.3^{\circ} \times\right.$ $1.3^{\circ}, 20 \%$ Michelson contrast) was presented at the center of gaze and instructed the monkey to direct attention to a position outside the RF of the neuron under study. To instruct the monkey to allocate attention inside the RF, a triangle cue $\left(1.3^{\circ} \times 1.3^{\circ}, 20 \%\right.$ Michelson contrast $)$ was shown instead. After a variable delay, two bars $\left(2.2^{\circ} \times 0.3^{\circ}\right)$ were simultaneously presented, one inside and one outside the RF, and they were placed in symmetrical positions with respect to the fixation spot and at equal distance from it. The bars were shown at two possible orientations (the optimal and one suboptimal for the neuron), independently chosen on a random basis at the two locations. To earn a juice reward, the monkey had to discriminate the orientation of the bar at the cued location by turning a lever in the appropriate direction. The bar inside the RF was displayed at seven or nine positive contrast levels (from 2.5 to $94 \%$ Michelson contrast) selected randomly on each trial; additionally, on some trials, a zero contrast bar was displayed inside the RF and the monkey was rewarded for any response, and we used these trials to establish baseline activity of the neurons. The bar outside the RF was of constant contrast (20\%) for one animal (monkey F), whereas it varied between 5 and $60 \%$ Michelson contrast for the other animal (monkey T); in the latter case, contrast at the two locations was independently selected on a random basis. We adopted such a protocol for the second animal to have the animal perform a nearly identical task in the two attentional conditions. Crucially, this did not lead to any appreciable difference between the two monkeys in terms of behavioral performance or neuronal responses (see Results). Moreover, to exclude the possibility that the broadband spatial frequency content or the overall increase in luminance might affect results from the main experiment, we also performed a control experiment using Gabor patches $(\sigma=0.4$, mean luminance of $42 \mathrm{~cd} / \mathrm{m}^{2}$ ) shown at the same contrast levels. Importantly, in both experiments, we recorded responses from $\geq 12$ repetitions (typically 16) for each stimulus condition.

Fitting procedures. Single-neuron CRFs were determined for each cell using a non-weighted, least-squares fitting procedure (MATLAB, Curve Finder Tool or CFTool). The mean firing rate of each neuron was fitted to an extension of the traditional Naka-Rushton function (Albrecht and Hamilton, 1982), namely the Peirce function (Peirce, 2007): Response $=R_{\max } \frac{C^{n}}{C_{50}^{s n}-C^{s n}}+R_{0}$, where $R_{\max }$ is the firing rate at which the curve asymptotes (note that it corresponds to the maximal response for monotonic functions), $R_{0}$ is the undriven activity, $C_{50}$ is the semisaturation contrast, i.e., the contrast value needed to reach half of the maximal response rate, the exponent $n$ represents the slope of the curve, and $s$ is the suppressive exponent. When $s$ assumes a value of 1 , the function corresponds to the traditional Naka-Rushton function, whereas higher values correspond to non-monotonic patterns. To be conservative, we considered a cell to be non-monotonic when $s>1.1$ and used additional analyses to check for the reliability of cell characterization (see below). We evaluated the goodness of fit by calculating the $R^{2}$ value ( 1 - SSE/SST, where SSE is the sum of squares error, and SST is the sum of squares totals); results were described and further analyzed only for well fitted cells $\left(R^{2}>0.7\right)$. We also derived a series of additional parameters of direct interest to describe the shape of CRFs. Percentage of response suppression was calculated as the difference between maximal response for any value of contrast and the response at maximum contrast, as analytically estimated from the curve, normalized to baseline firing $\left(R_{0}\right)$. For non-monotonic cells, we calculated peak contrast as the contrast at which the maximal response occurred and the bandwidth as the difference between the two contrast levels at which the response was $3 / 4$ of the maximal response, corresponding to $25 \%$ inhibition; note that a minority of non-monotonic cells showed an inhibition $<25 \%$, and their bandwidth could not be measured.

With the aim of checking the reliability of cell characterization, in addition to the Peirce function, two alternative models were tested. To specifically describe monotonic CRFs, the mean firing rate of each neuron was fitted to the traditional Naka-Rushton function (Albrecht and 
A

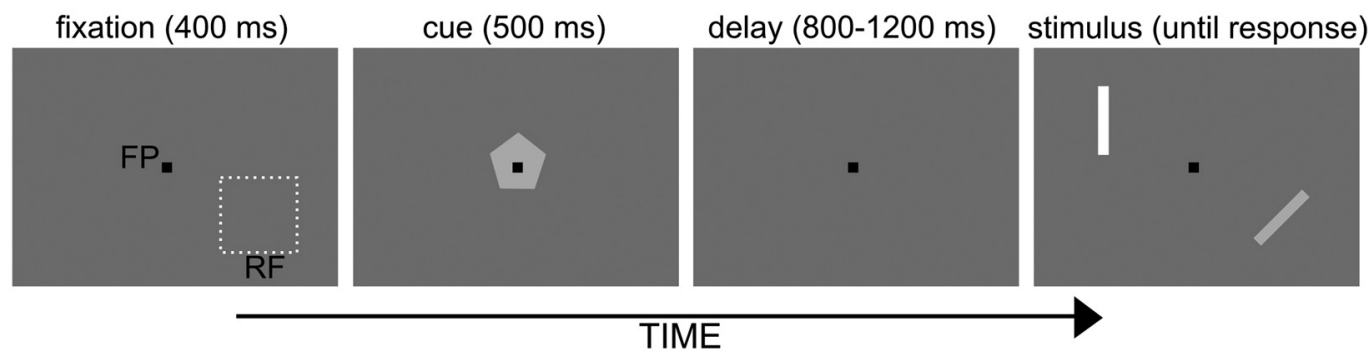

B

ACCURACY

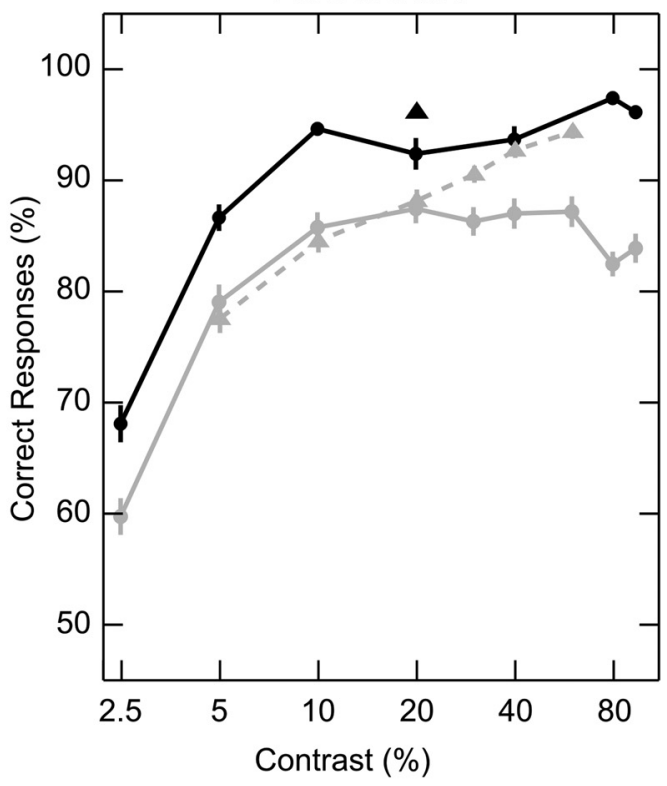

SPEED

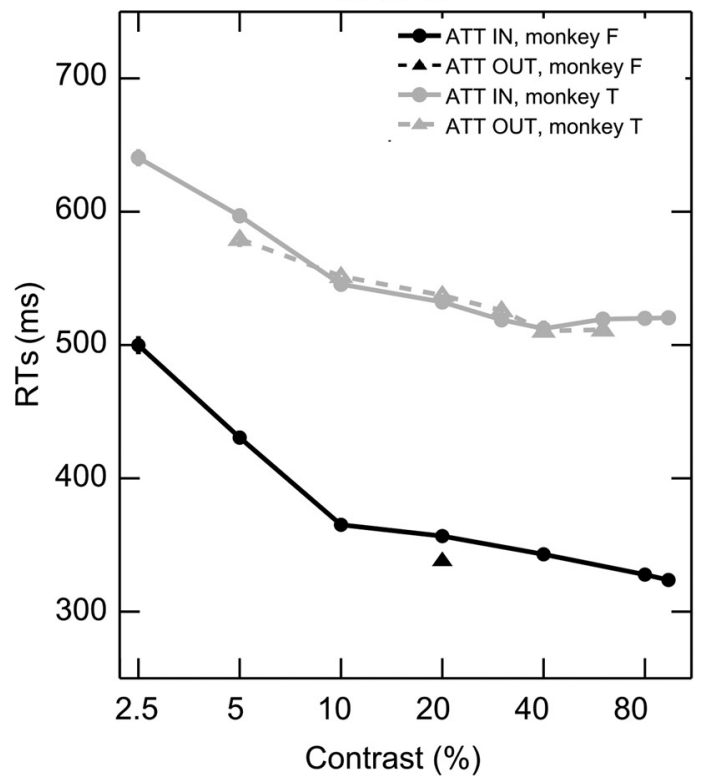

Figure 1. Experimental paradigm and task performance. $A$, Behavioral task. The panels illustrate the temporal sequence of events within an example trial. The animal was trained to perform orientation discrimination on a bar stimulus located outside the RF of the neuron (top left quadrant); meanwhile, a bar was also displayed inside the RF at different contrast levels in two possible orientations. FP is the fixation point; RF indicates the classical RF; the pentagon represents the cue stimulus instructing the animal to allocate attention outside the RF; bars represent the stimuli to be discriminated; labels indicate the duration of each event in milliseconds. Note that the background is shown in gray for illustrative purposes (real luminance value, $\left.2.49 \mathrm{~cd} / \mathrm{m}^{2}\right) . \boldsymbol{B}, \mathrm{Accuracy}$ (percentage of correct responses) and mean reaction times (RTs) as a function of contrast (percentage Michelson contrast) are represented in the left and right, respectively. Solid and dashed lines, respectively, depict accuracy (or reaction times) for the orientation discrimination task performed inside and outside the RF of the neuron under study, separately for monkey $F$ (in black) and monkey $\mathrm{T}$ (in gray). Only one data point is shown for monkey $\mathrm{F}$ in the attention-outside condition because the contrast was set to be constant (20\%).

Hamilton, 1982): Response $=R_{\max } \frac{C^{n}}{C_{50}^{n}-C^{n}}+R_{0}$, where $R_{\max }$ is the maximal mean firing of the neuron, $R_{0}$ is the undriven activity, $C_{50}$ is the semisaturation contrast, i.e., the contrast value needed to reach half of the maximal response rate, and the exponent $n$ represents the slope of the curve. To describe non-monotonic CRFs, we also performed fitting procedures using a skewed Gaussian function (Lisberger and Movshon, 1999): Response $=R_{\max } \exp \left(-1 / 2\left(\frac{\sigma}{\log \frac{C}{C_{p}}}+\right.\right.$ sk $\left.)\right)+R_{0}$, where $R_{\max }$ is the peak height, $C_{\mathrm{p}}$ is the contrast value for which the peak occurs, $\sigma$ is the peak width, sk is the skewness, and $R_{0}$ is the baseline activity. In this case, we established the proportion of non-monotonic cells through the comparison between the Naka-Rushton and the Gaussian function, respectively suitable to describe the traditional and the non-monotonic CRFs. We used several methods to evaluate the goodness of fit and compare the efficiency of the two functions in describing single-cell CRFs in our sample. We considered a cell as being non-monotonic when it was better fitted by the Gaussian function and monotonic when it was better fitted by the Naka-Rushton function. As a first step, we chose Aikake's information criterion (AIC; Motulsky and Christopoulos, 2004) for model comparison, because it is one of the most widely used methods. The AIC value was calculated for each equation as $n \log (\mathrm{RMS})+2 p$, where $n$ represents the number of data points being modeled, $p$ repre- sents the number of parameters in the model, and RMS is the root mean square error of the model fit. The model with the smallest value of AIC is most likely to be correct. We computed the probability of choosing the correct model by the following equation: $e^{-0.5 \Delta} /\left(1+e^{-0.5 \Delta}\right)$ where $\Delta$ is the difference between AIC scores. We also used a related method, the Bayesian information criterion (BIC), which is identical except that the penalty term is $\log (n) p$ instead of $2 p$, thus being more stringent. An alternative method, referred to as the normalized $\chi^{2}$ by Cavanaugh et al. (2002), takes the $\chi^{2}$ error term $\Sigma_{i} \frac{\left(e_{i}-o_{i}\right)^{2}}{\sigma_{i}^{2}}$, where $i$ is the index of this particular contrast level, $e$ is the expected response at this contrast level given the current model parameters, $o$ is the observed response, and $\sigma^{2}$ is the trialwise variance in responses at this contrast. The $\chi^{2}$ error term is then normalized by the degrees of freedom of the model. As with the AIC, the best fitting and most efficient model is taken to be that with the lowest normalized $\chi^{2}\left(\chi_{\mathrm{N}}^{2}\right)$ value; also this method inflicts a more stringent penalty on models with additional parameters. We finally evaluated the adjusted $R^{2}$ value as $1-\frac{\frac{\mathrm{SSE}}{n}-(p+1)}{\frac{\mathrm{SST}}{n-1}}$, where SSE is the sum of squares error, SST is sum of squares totals, and $(p+1)$ penalizes the number of parameter. Both the Peirce function and the combination of 
Naka-Rushton and skewed Gaussian functions provided a good quantitative description of the recorded neurons. However, we chose the Peirce function as the standard approach because of its ability to characterize any of the cells without the need of preclassifying them.

Analysis of temporal dynamics. We quantified the degree to which the response of neurons rises monotonically with respect to contrast using a dimensionless monotonicity index (MI) (Ledgeway et al., 2005) calculated as follows: $1-\left(R_{\max }-R_{100}\right) /\left(R_{\max }-R_{0}\right)$, where $R_{\max }$ is the maximum response of the neuron, $R_{100}$ is the response to the maximal contrast used, and $R_{0}$ is the response to the null stimulus. MI assumes the value of 1 when the response is monotonically increasing (i.e., the response to the highest contrast is the maximal response of the neuron); it is smaller than 1 when the pattern is non-monotonic (i.e., the response to the highest contrast is not the maximal response of the neuron). Note that MI can assume the value of -infinite when $R_{\max }$ equals $R_{0}$ or it is undefined when $R_{\max }, R_{100}$, and $R_{0}$ are equal; such values were not included in the average calculation (in the poststimulus epoch their incidence was marginal: $10 \%$ on average). To quantify the effects of stimulus orientation on neuronal response, we computed the orientation index (OI) as follows: $\frac{\left(R_{\text {pref }}-R_{\text {unpref }}\right)}{\left(R_{\text {pref }}+R_{\text {unpref }}\right)}$, where $R_{\text {pref }}$ is the response of the neuron to the preferred orientation, and $R_{\text {unpref }}$ is the response to the unpreferred stimulus; the percentage orientation selectivity was calculated as $100 \frac{2 \mathrm{OI}}{(1-2 \mathrm{OI})}$. Note that both MI and OI will assume a value of $\sim 0$ before stimulus onset; in fact, at that time, the maximum response of the neuron and the response to the maximal contrast, for MI, and the response to the preferred and unpreferred orientations, for OI, do correspond to baseline activity, thus leading to a difference approximately equal to 0 .

To achieve a good level of temporal resolution, we calculated mean firing rate, and consequently both indices, in 200 overlapping time windows (20 ms width, $1 \mathrm{~ms}$ shift). Trials were aligned with respect to stimulus onset or to neuronal response onset. In the latter case, latency of the neuronal response for each contrast level was used as the starting point of the first time window. Operationally, we constructed a peristimulus time histogram (PSTH) and smoothed it with a Gaussian filter $(\sigma=8 \mathrm{~ms})$; we then defined and calculated latency as time to half the peak of the response waveform (Gawne et al., 1996).

To illustrate the temporal dynamics of CRFs (see Fig. 6), we used mean firing rate calculated in 16 partially overlapping time windows $(50 \mathrm{~ms}$ width, $10 \mathrm{~ms}$ shift, covering a total interval of $200 \mathrm{~ms}$ ), starting from response onset, as defined previously.

To compare cell behavior between the early and late phases of visual response, we selected the two windows with the most divergent pattern of monotonicity (0-50 and $100-150 \mathrm{~ms}$ after response onset) to perform fitting procedures.

\section{Results}

We trained two adult macaque monkeys to discriminate the orientation of achromatic bar stimuli in return for juice reward (see Materials and Methods; Fig. 1A). Because we also planned to characterize the influence of spatial attention on neuronal CRFs in macaque area $\mathrm{V} 4$ - an aspect of the study that is not reported here-the general approach was chosen to be compatible with this goal. As illustrated in detail in Materials and Methods, in separate blocks, the animals were instructed to direct attention to either of two bars simultaneously presented on the screen, one inside and one outside the RF. The bar inside the RF was displayed at various contrasts selected randomly on each trial. The one outside the RF was of constant contrast (20\%) for one animal, whereas it varied between 10 and $80 \%$ for the other animal; in the latter case, contrast at the two locations was independently selected on a random basis. After the monkeys reached a high level of performance at the task (Fig. $1 B$ ), we recorded responses of V4 neurons to different contrasts; 332 cells (172 from monkey $\mathrm{F}$ and 160 from monkey $\mathrm{T}$ ) were selected for in-depth analysis on the basis of the quality of recordings. In what follows, we will mainly report on responses elicited by an optimally oriented stimulus with attention allocated outside the RF, except when otherwise stated.

\section{Heterogeneous CRFs in V4}

Figure $2 A$ shows the PSTHs of two representative neurons. Both neurons display a decrease in latency with increasing contrast and a phasic response to the stimulus, followed by rapid adaptation, reaching a plateau $\sim 150-200 \mathrm{~ms}$ after stimulus onset. Despite the similarity in their transient behavior, the two neurons show different CRFs, i.e., a monotonically increasing (top panels) and a non-monotonic (or bandpass; bottom panels) profile, respectively. In the top example, as contrast increases, the PSTHs scale upward, whereas in the bottom example, the maximal response clearly occurs for intermediate levels of contrast.

To characterize quantitatively neuronal responses to varying contrast levels, we calculated mean firing rate in a $100 \mathrm{~ms}$ time window starting $40 \mathrm{~ms}$ after stimulus onset (Fig. $2 \mathrm{~A}$, bottom right), according to the average response latency to the highest contrast in the sample of recorded neurons. We then determined single-neuron CRFs by fitting the Peirce function (Peirce, 2007), an extension of the traditional Naka-Rushton function (Albrecht and Hamilton, 1982), which is able to accommodate for both monotonic and non-monotonic patterns of response by virtue of a suppressive exponent (see Materials and Methods). For a suppressive exponent equal to 1 , the cell is monotonic, whereas for higher values, it is non-monotonic. The sample of recorded neurons showed highly heterogeneous CRFs, including increasing, saturating, and non-monotonic patterns (Fig. $2 B$ ). Consistently, the distribution of the suppressive exponent (Fig. $3 A$, left) showed a substantial proportion of cells showing varying degrees of suppression at high contrasts $(s>1)$ in addition to traditional monotonic saturating cells $(s \leq 1)$. To estimate the attenuation of response occurring at the maximal contrast, we calculated the percentage of response suppression from the fitted data (see Materials and Methods): half of the total well fitted cells showed a degree of attenuation $>10 \%$ (Fig. $3 A$, right). Figure $3 A$ confirms that contrast coding in the population of recorded cells was highly heterogeneous and distributed along a continuum, ranging from increasing CRFs to narrow bandpass filter tuning profiles in response to contrast. To provide a systematic description of parameters characterizing monotonic versus nonmonotonic cells, we set a conservative criterion to diagnose selective tuning for contrast, corresponding to a suppressive exponent $(s)$ of 1.1 (see Materials and Methods). Accordingly, selective tuning for contrast was diagnosed in $31 \%$ of the total neurons. Additional analyses and considerations over classification issues confirmed the existence of a reliable and consistent proportion of cells showing selective tuning for contrast in the population (see below). Importantly, the incidence of contrast-selective cells was comparable between the two monkeys (34 and 28\%, respectively, in monkeys $\mathrm{F}$ and $\mathrm{T}$ ), excluding an account of the observed phenomenon in terms of a contrast assimilation effect. In fact, although the contrast of the bar outside the RF was constant for one monkey (monkey F), it varied randomly across trials for the other (monkey $\mathrm{T}$ ). Compatible results were obtained under conditions in which attention was allocated inside the RF of the neurons under study (36\% of contrast-selective neurons, on average, across the two animals) and also in a passive fixation condition in which only one stimulus appeared on the screen $(34 \%$ of contrast-selective neurons, 37 of 109 well fitted cells). These 
A
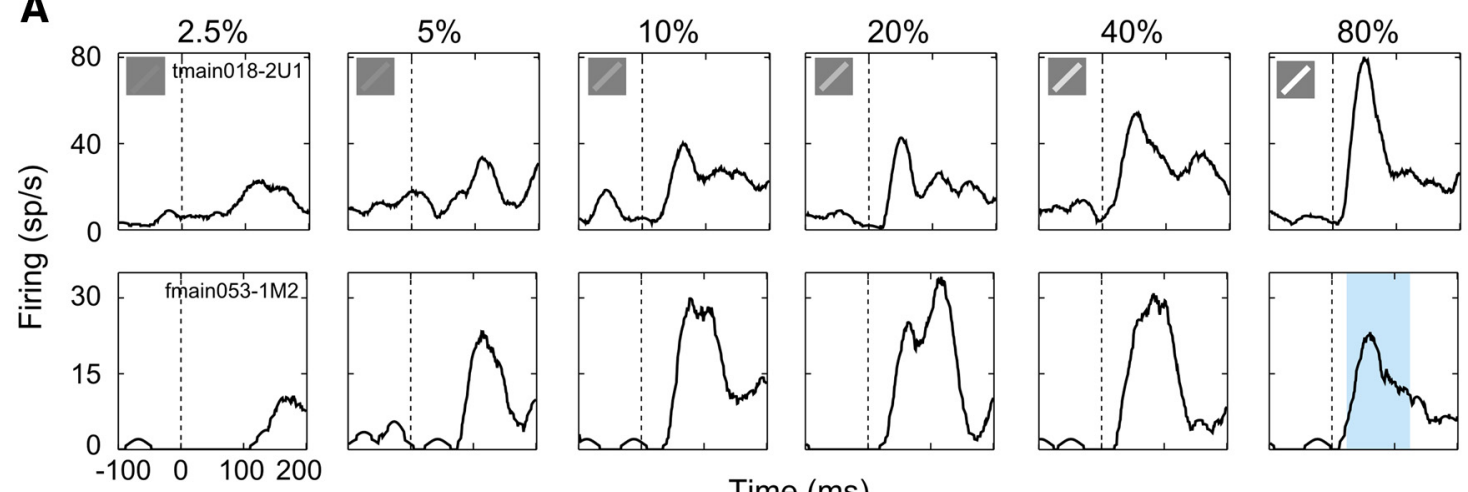

Time (ms)
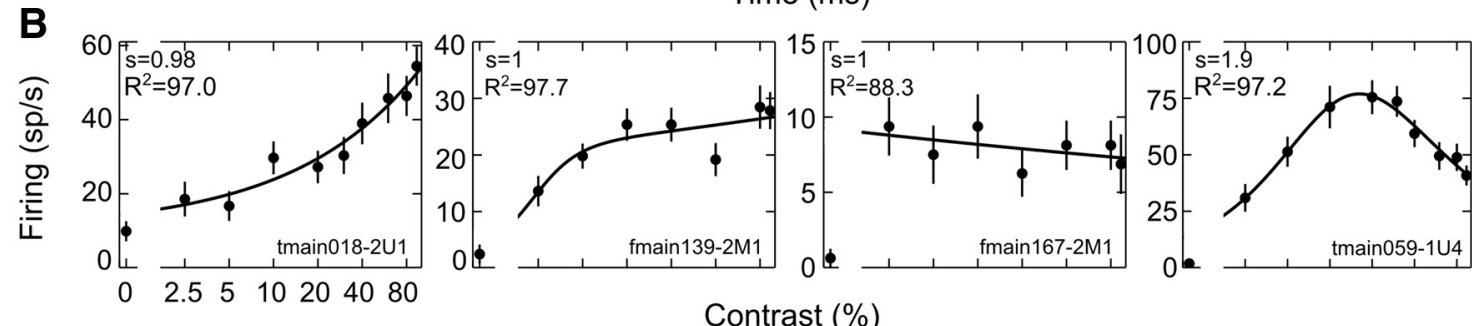

Contrast (\%)

Figure 2. Response of V4 neurons to luminance contrast. A, PSTHs. PSTHs are plotted for six logarithmically spaced levels of contrast and aligned with stimulus onset for a monotonically increasing (top row) and a contrast-selective (bottom row) single-cell example. The light blue shaded area represents the $100 \mathrm{~ms} \mathrm{time} \mathrm{window} \mathrm{used} \mathrm{for} \mathrm{some} \mathrm{of} \mathrm{the} \mathrm{analyses} \mathrm{to} \mathrm{calculate} \mathrm{mean} \mathrm{firing}$ rate of the recorded cells. $\boldsymbol{B}$, Monotonic increasing, monotonic saturating, non-modulated, and selective single-cell examples. Mean firing rate (spikes per seconds) is plotted as a function of percentage Michelson contrast. Each point represents the average of $\geq 12$ stimulus presentations, along with its SEM (vertical lines). Solid lines depict the best fitted curve provided by the Peirce equation. $s$ represents the value of the suppressive exponent given by the fitting procedures; note that this value is subsequently used to label cells as monotonic ( $s \leq 1.1)$ or selective ( $s>1.1$; see Results).

results rule out accounts of the phenomenon in terms of attentional effects. Finally, similar results were obtained using Gabor stimuli instead of bars (28\% of contrast-selective neurons, 37 of 132 well fitted cells; see Materials and Methods), thus excluding a potential influence of the broadband spatial frequency content of the bar stimuli or of variations in mean luminance.

\section{Quantitative properties of monotonic and selective CRFs}

Monotonic $(s \leq 1.1)$ and selective $(s>1.1)$ cells were examined separately. For traditional monotonic cells (Fig. 3B), the semisaturation contrast of the neurons, as described by the $C_{50}$ parameter (left panel), clustered around very low contrast levels (mean \pm SEM, $5.2 \pm 0.3$ ), reflecting high contrast sensitivity across the neuronal population. Likewise, the slope of the curves tended to be very steep (mean \pm SEM, $6.5 \pm 0.3$; Fig. 3B, right), reflecting high contrast discriminability within the dynamic range. Such behavior implies a great capacity to detect very low contrast stimuli but a limited capacity to discriminate among high contrast levels. Contrast sensitivity in monotonic V4 cells thus appears to be higher than reported in previous studies (Cheng et al., 1994; Williford and Maunsell, 2006), possibly caused by a number of factors, including the type of stimulus and the type of analysis. For selective cells, peak contrast and bandwidth were calculated from the fitted data (see Materials and Methods). Peak contrast spanned the whole range of contrasts (Fig. 3C, left), implying that different cells are specifically responsible for coding different levels of contrast. The full bandwidth of selective neurons ranged from 0.04 to $1.4 \mathrm{log}$ units (Fig. 3C, right), reflecting a variable degree of selectivity across the population. The two patterns of response, namely the traditional monotonic and the non-monotonic profiles, are likely to play a distinct role in contrast coding (see below). In particular, neurons characterized by bandpass tuning are well suited to encode luminance contrast in a way similar to what other neurons do in relation to other low-level visual features, such as color and orientation.

Consistency of classification of monotonic and selective CRFs To assess the reliability of the classification approach used to unveil the existence of distinct subpopulations of monotonic and non-monotonic cells, we applied alternative and well established methods (see Materials and Methods) for modeling and classifying cells. Each and every classification approach we tested revealed the existence of a consistent fraction of non-monotonic CRFs (Table 1).

To further strengthen the idea that cells classified as nonmonotonic according to the suppressive exponent of the Peirce function were indeed tuned for contrast, we fitted data with a skewed Gaussian function (see Materials and Methods), which is typically used to describe selectivity tuning. We compared fitting results obtained from both equations using AIC (see Materials and Methods) so as to determine which model was more likely to be the correct one for the given cell. The scatter plot in Figure $4 \mathrm{~A}$ depicts AIC values for the Gaussian function and the Peirce function. Interestingly, the comparison between these two functions revealed that non-monotonic cells were fitted almost equally well with the Gaussian function (median explained variance, $90.54 \%$ ). Specifically, $57 \%$ of the cells were better fitted by the Peirce function and $43 \%$ by the Gaussian function. Note that points lie very close to the diagonal and the posterior probability of having chosen the right model is quite low (color coding). As evident in the two single-cell examples shown in Figure $4 B$, the two models describe non-monotonic patterns almost equally well; note that the selected examples are two of the most extreme cases, namely among the more distant points from the diagonal (Fig. 4A, black points). 
The Gaussian function provided a very good model for non-monotonic CRFs (Fig. $4 A, B)$, whereas the traditional NakaRushton function (Albrecht and Hamilton, 1982; see Materials and Methods) is known to be a good descriptor of saturating monotonic CRFs. We tested the efficiency of these two alternative models in describing and discriminating between monotonic and non-monotonic patterns of response to contrast. The scatter plot in Figure $4 C$ depicts AIC values for the Naka-Rushton function and the Gaussian function. Because low values of AIC indicate that the regression line properly fits the data, points on the left of the diagonal correspond to neurons better fitted by the Naka-Rushton function (monotonic CRFs), whereas points on the right of the diagonal correspond to neurons better fitted by the Gaussian equation (non-monotonic CRFs). Interestingly, data are distributed almost continuously in the range between monotonic and non-monotonic CRFs. Despite this, most of the data points are not lying on or near the diagonal, and posterior probabilities to have chosen the right model are quite high (color coding: pure red and blue points). Note that there is a substantial proportion of data points lying on the right of the diagonal (21\%), meaning that the CRFs of the corresponding cells are better fitted by the Gaussian equation and are therefore non-monotonic according to this method. As evident in Figure 4D, with this approach, monotonic saturating cells are better described by the traditional Naka-Rushton function, whereas nonmonotonic patterns are better described by the Gaussian function.

In addition to AIC, we compared the Naka-Rushton and Gaussian functions using different approaches (see Materials and Methods), namely AIC, BIC, adjusted $R^{2}$, and $\chi_{\mathrm{N}}^{2}$; results are summarized in Table 1 . All the comparisons revealed that two different patterns of contrast response, namely monotonic and non-monotonic, emerge in our sample of neurons. The small differences in classification rate obtained with different approaches reflect the continuum in CRF profiles between highly monotonic and non-monotonic profiles; in other words, V4 neurons showed very heterogeneous patterns of response to contrast, including increasing, perfectly saturating, weakly non-monotonic, and Gaussian-like profiles.

\section{Temporal dynamics of CRFs}

To study the temporal dynamics of contrast encoding in area V4, we first calculated population PSTHs (Fig. 5). As can be seen in Figure $5 A$, the average latency of response decreased significantly as contrast increased, in line with previous reports (Albrecht, 1995; Gawne et al., 1996; Lee et al., 2007). As shown in the figure, the average population response approached saturation at $40 \%$ contrast, reflecting a monotonic behavior at the peak. Interest-

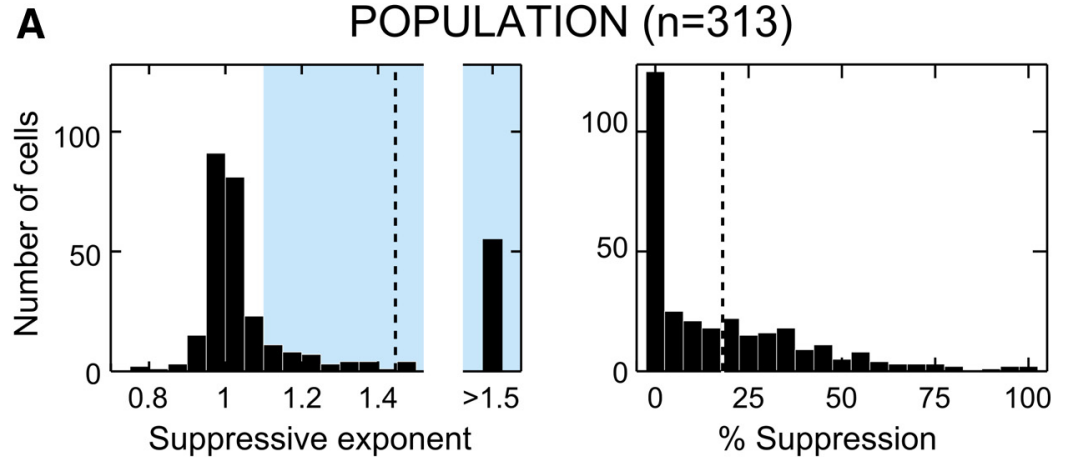

B MONOTONIC CRFs $(\mathrm{n}=216)$
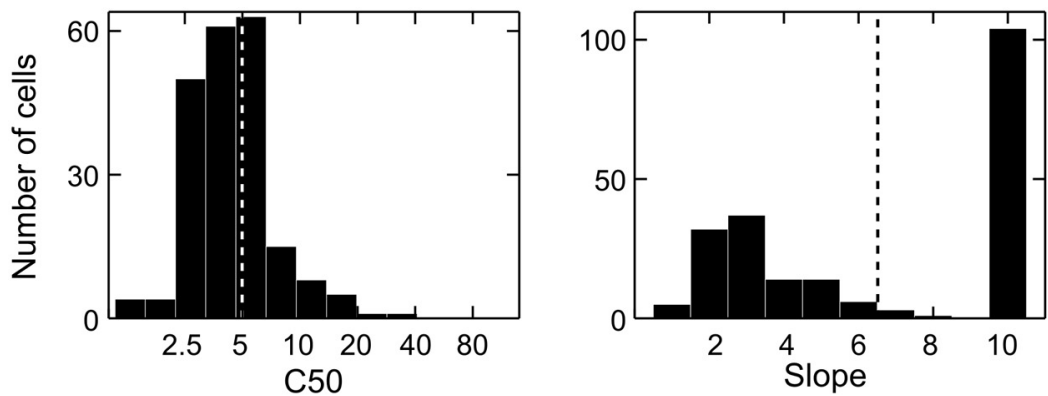

SELECTIVE CRFs $(\mathrm{n}=97)$
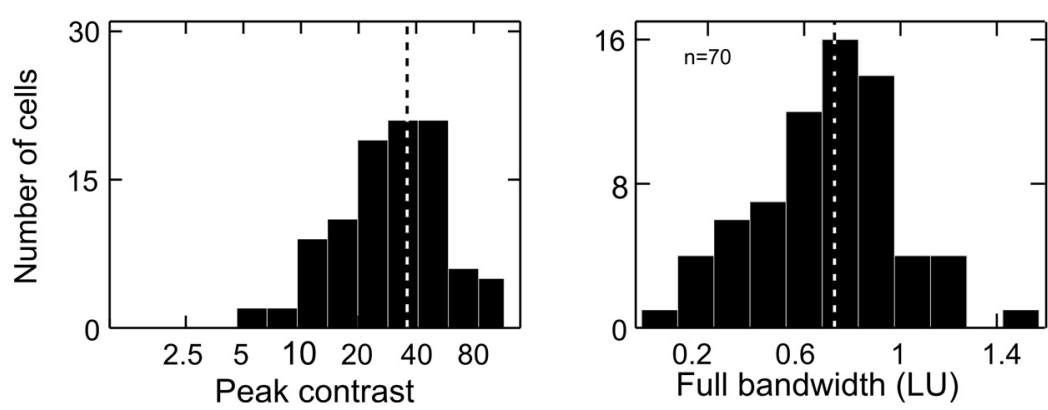

Figure 3. Population and subpopulation properties of CRFs. $A$, Population. The panels show the distribution of the suppressive parameter ( $(s)$, as given by each fitted Peirce function (median explained variance, $91.4 \%$ ), and the percentage of suppression for each CRF, as derived from the Peirce function; the parameters are reported for all the well fitted neurons. The vertical dotted lines represent the average value of the depicted parameter. The light blue shaded area highlights the cells considered to be selective for contrast $(s>1.1)$. $\boldsymbol{B}$ ter distribution for monotonic cells. The panels represent the distributions of $C_{50}$, expressed in percentage Michelson contrast, and slope for the population of monotonic cells $(s \leq 1.1)$. Conventions as in $\boldsymbol{A}$. C, Parameter distribution for selective cells. The panels represent the distributions of peak contrast and full bandwidth for the population of selective cells $(s>1.1)$, expressed in percentage Michelson contrast and log units (LU), respectively. Note that the bandwidth is reported for the selective cells showing at least $25 \%$ of inhibition (see Materials and Methods, Fitting procedures). Conventions as in $\boldsymbol{A}$.

Table 1. Model comparison and cell classification: consideration over the proportion of cells showing non-monotonic tuning for contrast

\begin{tabular}{|c|c|c|c|c|c|c|c|c|c|c|}
\hline \multirow[b]{2}{*}{$(n=332)$} & \multicolumn{2}{|c|}{$\begin{array}{l}\text { Suppressive } \\
\text { exponent }\end{array}$} & \multicolumn{2}{|l|}{ AIC } & \multicolumn{2}{|l|}{$\mathrm{BIC}$} & \multicolumn{2}{|c|}{$\begin{array}{l}\text { Adjusted } \\
R^{2} \\
\end{array}$} & \multicolumn{2}{|l|}{$\chi_{N}^{2}$} \\
\hline & M & NM & $M$ & NM & $M$ & NM & M & NM & $M$ & NM \\
\hline & 227 & 105 & 263 & 69 & 267 & 65 & 164 & 168 & 210 & 122 \\
\hline Percentage of cells & 68 & 32 & 79 & 21 & 80 & 20 & 49 & 51 & 63 & 37 \\
\hline
\end{tabular}

The number and percentage of cells classified as monotonic (M) and non-monotonic (NM) are reported based on the suppressive exponent value and other classification approaches based on the evaluation of the goodness of fit corrected for the number of parameters in the model (see Materials and Methods, Model comparison).

ingly, the population response showed a marked drop immediately subsequent to the initial transient increase in firing for all tested contrasts except the lowest, implying that higher contrasts engage proportionally greater suppressive effects compared with 
A
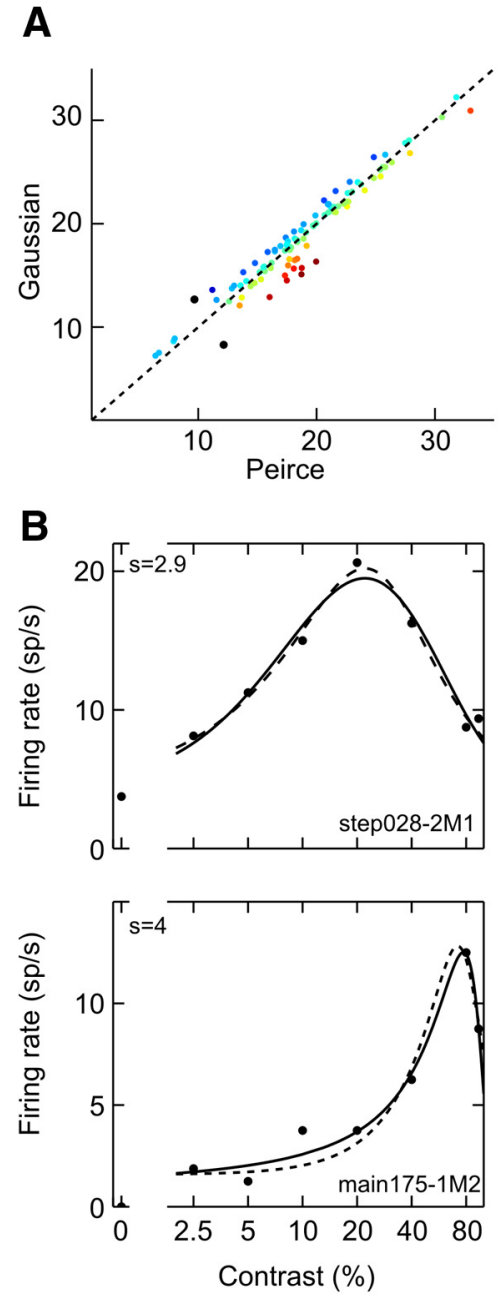

C

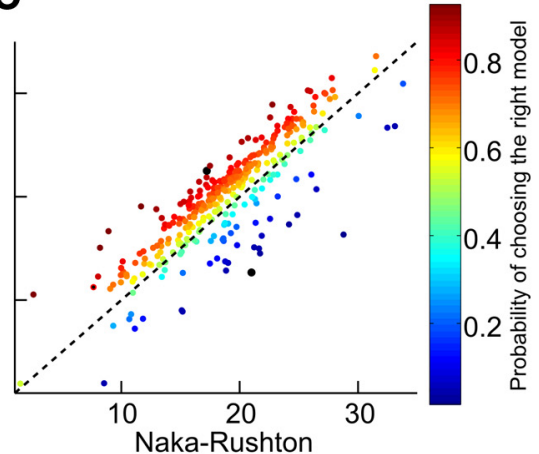

D
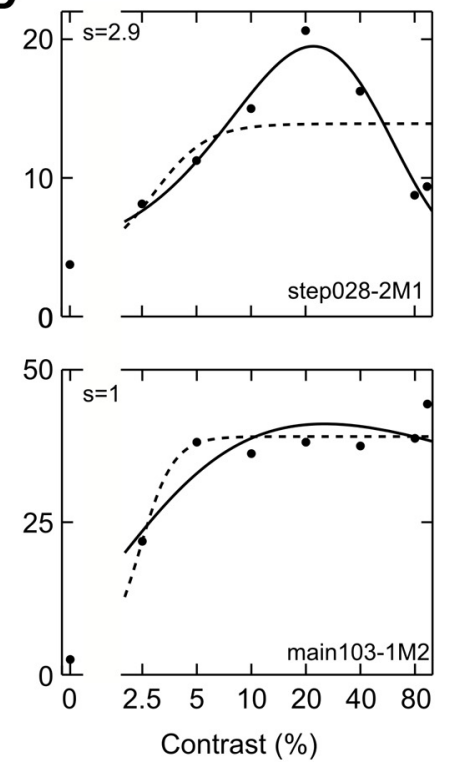

Figure 4. Consistency of classification of monotonic and selective CRFs. A, Peirce versus Gaussian function. The scatter plot depicts AIC values for the Gaussian function and the Peirce function fitted to the cells classified as selective for contrast according to the suppressive exponent ( $s$ ) of the Peirce function (see Results, Heterogeneous (RFs in V4). Each data point represents a fitted neuron. The color of each point depicts the posterior probability to belong to one or the other of the two models; black dots represent the two neurons shown in $\boldsymbol{B}$. Note that the lower the AIC value, the better is the fitting. $\boldsymbol{B}$, Non-monotonic single-cell examples. Responses of two representative V4 neurons to bars of different contrast are shown. Mean firing rate (spikes per second) is calculated in a 100 ms time window starting at $40 \mathrm{~ms}$ after stimulus onset and is plotted as a function of percentage Michelson contrast. Each point represents the average of $\geq 12$ stimulus presentations; the value of the suppressive exponent (s) calculated by fitting the Peirce function is reported for each cell. Lines depict the best fitted curve provided by the Peirce equation (dashed line) and the Gaussian equation (solid line). Note that the selected examples are two of the most extreme cases (more distant from the diagonal) in the scatter plot (A). C, Gaussian versus Naka-Rushton function. The scatter plot depicts AIC values for the NakaRushton function and the Gaussian function (convention as in $\boldsymbol{A}$ ); black dots represent the two neurons shown in $\boldsymbol{D}$. Note that low values of AIC indicate that the regression line properly fits the data. $\boldsymbol{D}$, Monotonic saturating versus non-monotonic single-cell examples. Responses of two representative V4 neurons (reported in black in $\boldsymbol{C}$ ) are shown (conventions as in $\boldsymbol{B}$ ). Lines depict the best fitted curve provided by the Gaussian equation (solid line) and the Naka-Rushton equation (dashed line).

lower contrasts; in other words, $\sim 200 \mathrm{~ms}$ after stimulus onset, neuronal activity became nearly independent of contrast (except for the lowest contrast). Given that the population of recorded neurons included a substantial proportion of selective neurons (Fig. 3A), we considered these neurons separately (Fig. 5B). In the early phase after stimulus onset, the average response of this population of neurons reached a maximum at $40 \%$ contrast, whereas higher contrasts led to lower responses (darker lines). Again, a clear attenuation of firing occurred at $\sim 100-150 \mathrm{~ms}$ and was particularly strong for high contrasts, so that the average response at $\sim 200 \mathrm{~ms}$ after stimulus onset was highly similar across all contrasts. This pattern of results was even more pronounced in the subpopulation of those selective cells displaying maximal re- sponses for contrast values lower than 20\% (Fig. 5C). Here the average maximal response in the early phase occurred for $20 \%$ contrast, and the response became contrast independent at $\sim 150 \mathrm{~ms}$ after stimulus onset, although there was a small tendency for the highest contrast to produce the smallest response. It is worth noting that the leftward shift in the latency of response along the range of tested contrasts was equally evident for the selective cells (Fig. $5 B, C$ ). The results in Figure 5 suggest that, across the population, the degree of non-monotonicity increases over time.

To achieve fine-grain analysis of the temporal dynamics of neuronal responses, we calculated mean firing in 20 ms time windows shifted by $1 \mathrm{~ms}$. As a first step, we computed the MI (Ledgeway et al., 2005) within each $20 \mathrm{~ms}$ window. MI is defined as the ratio between firing rate enhancement (or reduction) at the highest contrast tested relative to the maximum response (at any contrast) and the difference between maximum response and the baseline (see Materials and Methods). As shown in Figure $6 A$, across the population, MI increased sharply in the first 15-20 ms after onset of the preferred stimulus (Fig. 6A, solid black line), reaching a maximum at $\sim 80 \mathrm{~ms}$. Monotonicity then decreased rapidly and reached a minimum at $\sim 150 \mathrm{~ms}$ after stimulus onset, meaning that the highest contrast triggered maximal suppression around this point in time. This timing is compatible with the intervention of a relatively slow inhibitory mechanism (see Discussion). After $150 \mathrm{~ms}$, MI showed a weak increase to then level off for the remainder of the trial. Importantly, the drop in monotonicity was in no way attributable to the high level of discharge rate induced by the optimally oriented stimulus. Indeed, the MI calculated for the nonpreferred orientation underwent an even greater reduction over time (Fig. 6A, black dotted line); the difference observed between the two orientations is likely linked to the concomitant increase in orientation selectivity (see Materials and Methods) over time at the population level (Fig. 6A, red line). Intriguingly, the temporal dynamics of orientation selectivity undergo a phase of increase that closely matches the timing of monotonicity reduction. This suggests important commonalities between the circuits and mechanisms mediating the emergence and refinement of both contrast selectivity and orientation selectivity, likely including a common inhibitory influence (see Discussion).

To rule out the possibility that the observed latency reduction with contrast (Fig. 5) could at least partly account for the observed reduction in MI, PSTHs were aligned with the latency of response, and mean firing rate was calculated in $20 \mathrm{~ms}$ time win- 

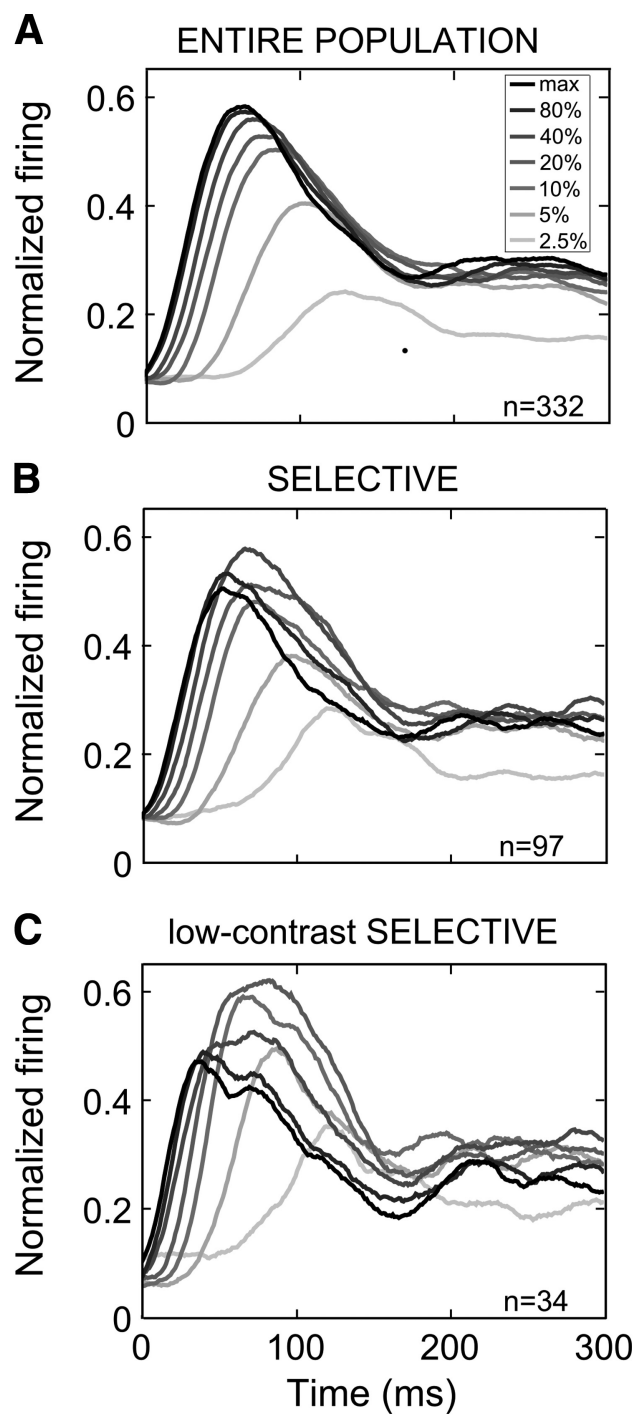

Figure 5. Population responses over time. Population PSTH for the preferred orientation is represented for each contrast level, aligned with stimulus onset (increasingly darker lines correspond to progressively higher contrasts). $\boldsymbol{A}$, Average normalized PSTH for the entire population of recorded cells. B, Population PSTH for selective cells. C, Population PSTH for selective cells showing peak responses at low contrast levels ( $<20 \%$ Michelson contrast). Note that, for illustration purposes, $40 \mathrm{~ms}$ bin width has been used throughout. As indicated by the leftward shift of the PSTH, average latency decreased significantly as contrast increased, and this was true regardless of whether we considered the entire population of recorded neurons $\left(F_{(6)}=\right.$ $126.82)$, the entire population of selective cells $\left(F_{(6)}=40.6\right)$, or the subpopulation of selective cells having a peak for $<20 \%$ contrast $\left(F_{(6)}=9.21 ; p \ll 0.01\right.$, 1-way ANOVA).

dows starting from response onset (see Materials and Methods; this approach was applied for all subsequent analyses). Crucially, with this approach and for both stimulus orientations, MI still showed a rapid increase at response onset $(\sim 0 \mathrm{~ms})$, followed by a progressive decrease and a minimum centered at $\sim 120 \mathrm{~ms}$ (Fig. $6 B)$, replicating the results in Figure $6 A$.

We then tested whether all single neurons showed a decrease in monotonicity over time by comparing MI values in two critical time windows (0-50 and 100-150 ms from response onset; Fig. $6 B)$. Across the population of recorded neurons, $63 \%$ of cells showed a decrease in monotonicity, whereas $29 \%$ of cells showed an increase in monotonicity ( $8 \%$ of the cells did not show any change in MI). Importantly, MI showed markedly diverging dynamics in these two subpopulations (Fig. 6C): whereas the for- mer was characterized by a strong reduction of average MI over time (Fig. $6 C$, solid line), the latter displayed on average a nearly flat temporal dependence (Fig. $6 C$, dotted line). Finally, to illustrate the dynamic changes of CRFs at the single-cell level, we plotted the mean firing rate calculated in partially overlapping time epochs as a function of time from response onset and contrast (see Materials and Methods). Figure 6, D and E, shows responses of two representative neurons in which CRFs changed from an initial monotonic function to a narrow bandpass tuning, whereas Figure $6 F$ shows a representative neuron whose CRF was simply rescaled over time, formally corresponding to a modest increase in monotonicity. Thus, it appears that the increase in non-monotonicity is a pervasive phenomenon in macaque area V4, although it does not affect all neurons in the sample.

Previous studies focusing on contrast response temporal dynamics applied very different approaches and found divergent results (Albrecht et al., 2002; Hu et al., 2011), with only Hu et al. (2011) showing an effect of time on the shape of CRFs. They focused their analysis on the first few tens of milliseconds after stimulus onset and found that CRFs changed from increasing to saturating. This is in line with our results, highlighting that both contrast saturation and contrast selectivity require time to develop and suggesting that a change in the shape of CRFs toward selective behavior probably occurs later in time, after the intervention of suppressive mechanisms (see Discussion). Critically, in both cited experiments, recordings were obtained in V1 of anesthetized animals. These two major differences with respect to our approach may have well prevented the occurrence of nonmonotonic tuning for contrast in those studies, with both the recording site and alertness/behavior of the animal potentially playing a crucial role.

Using best-fitting procedures, we directly and quantitatively compared, on a cell-by-cell basis, the two critical windows (Fig. $6 B)$ : an early window after response onset $(0-50 \mathrm{~ms})$, namely the one corresponding to the maximal degree of monotonicity, and a window in which maximal suppression for high contrasts occurred (100-150 ms), corresponding to the bandpass filtering stage. The proportion of cells displaying selective tuning $(s>1.1$; Fig. 7A, left) increased over time. Consistently, the percentage of suppression at high contrasts also increased considerably $(p<$ 0.01 ), from an average value of 16 to $38 \%$ (Fig. $7 A$, right). The distribution of $s$ in the two windows differed significantly $(p \ll$ 0.01 , Kolmogorov-Smirnov test), and the mean was significantly higher in the second window $(p \ll 0.01$, one-tailed, paired $t$ test). To further examine monotonic and selective cells separately, we concentrated on cells with the same overall type of behavior in both time windows to quantitatively assess changes in CRF parameters over time. Interestingly, in neurons showing monotonic CRFs in both time windows, we observed a significant increase in the slope from early to late epochs after response onset (Fig. $7 B$, right) but no reliable change in contrast sensitivity over time (Fig. $7 B$, left), corresponding to an enhanced capacity of the cell to discriminate contrasts within its dynamic range but also an overall narrowing of the range over which discriminative responses are possible. Of major interest, neurons with selective tuning in both time windows showed no obvious variation in peak contrast (Fig. $7 C$, left) but a considerable narrowing in bandwidth (Fig. 7C, right). The latter result reflects an increased capacity of the cells to selectively encode a specific range of contrast values. Figure $7 D$ shows four typical single-cell examples. In particular, the neuron shown in the top left panel maintains a monotonic pattern of response in both time epochs; the one in the bottom right panel maintains a selective behavior across ep- 
A
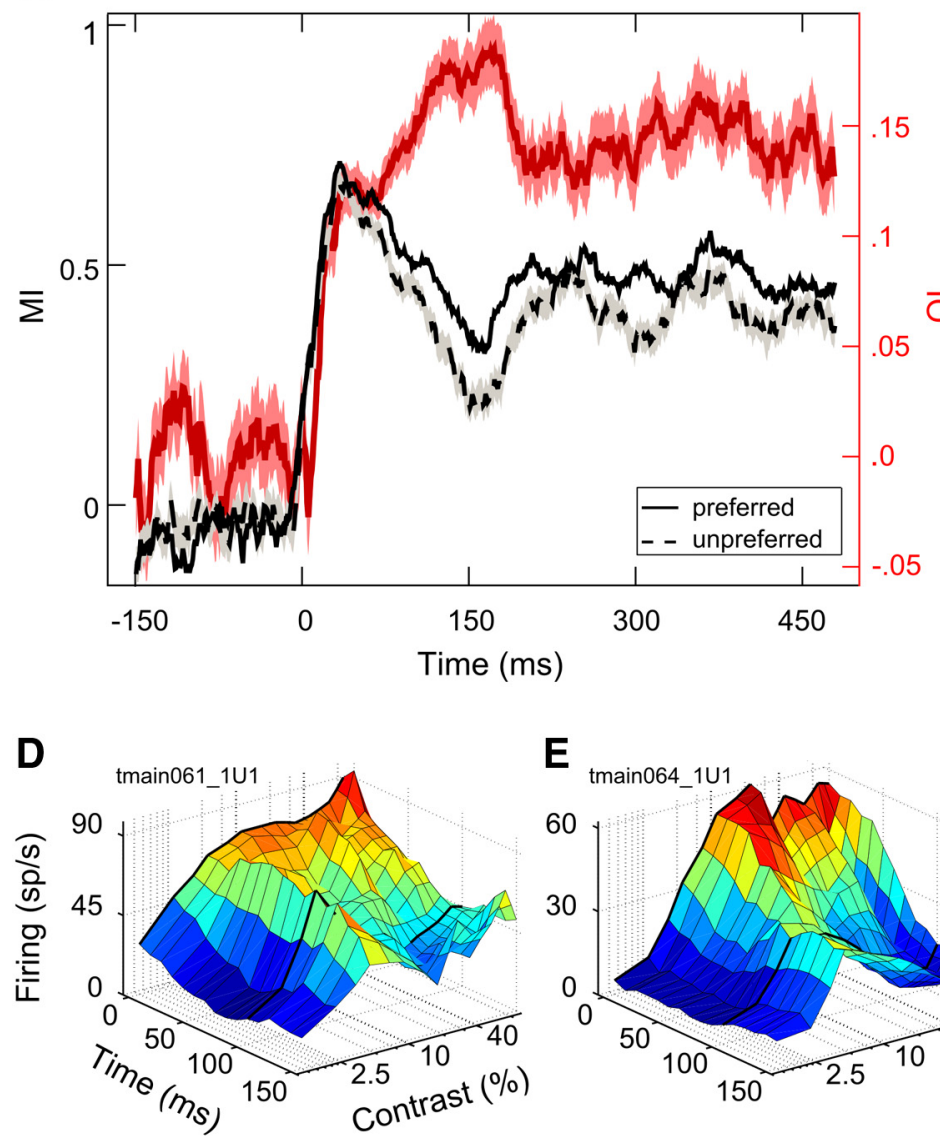

B

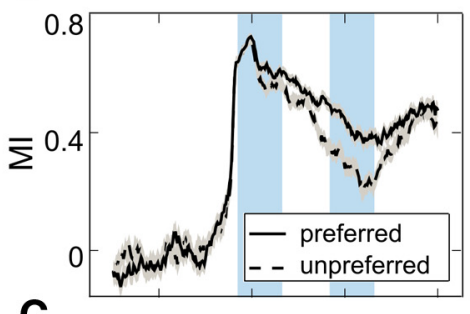

C

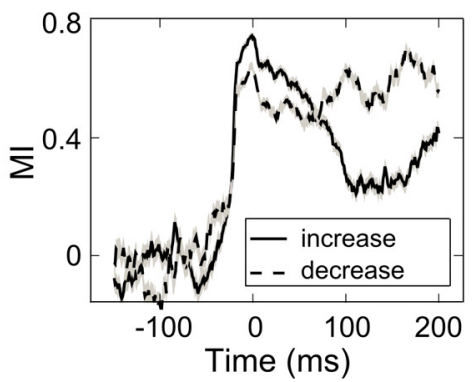

$\mathbf{F}$

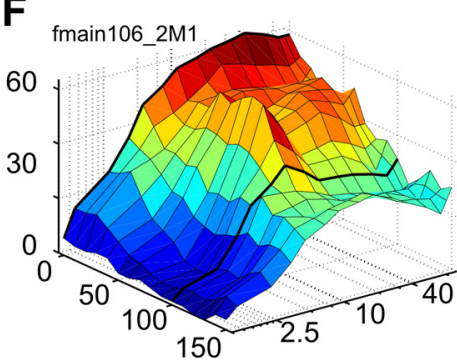

Figure 6. Changes in non-monotonicity over time. $\boldsymbol{A}$, Average Ml as a function of time, time locked to stimulus onset. Ml, calculated in 20 ms time windows, shifted by 1 ms and averaged across the population, is plotted as a function of time for both the preferred (solid line) and unpreferred (dotted line) orientation. The population average 0 ( (see Materials and Methods, Analysis of temporal dynamics) as a function of time is reported in red. Note that critical patterns for the two indices are coincident in time. $\boldsymbol{B}$, Average Ml over time, time locked to response onset (conventions as in $\boldsymbol{A}$ ). The light blue shaded areas represent the two 50 ms time windows of maximal interest. C, Average Ml over time for cells showing increasing or decreasing monotonicity. MI over time is reported separately for the subpopulation of cells with decreasing monotonicity $(n=209$, solid line) and the subpopulation of cells with increasing monotonicity ( $n=95$, dotted line) as calculated from the comparison between the time windows of maximal interest. $\mathbf{D}-\boldsymbol{F}$, Single-cell examples. Average firing rate [spikes per second (sp/s)] of three representative neurons plotted as a function of contrast and time for 16 overlapping time epochs ( $50 \mathrm{~ms}$ width, $10 \mathrm{~ms}$ shift); surface color turns from blue to red for progressively higher discharge rate. Note that, with reference to $\boldsymbol{C}$, single examples in $\boldsymbol{D}$ and $\boldsymbol{E}$ belong to the subpopulation of cells showing increasing non-monotonicity over time, whereas the example in $\boldsymbol{F}$ belongs to the subpopulation of cells with modestly decreasing nonmonotonicity over time.

ochs; finally, neurons in the other two panels undergo a transition from monotonic to bandpass tuning. Overall, $\sim 100-150 \mathrm{~ms}$ after response onset, V4 neurons in the population are able to segment sharply all the visible contrast range, with both steep high-pass and narrow bandpass contrast tuning.

To obtain a global appreciation of the properties of area V4 neurons in coding luminance contrast, we separately considered the populations of monotonic and selective cells at the maximal expression of their sensitivity or selectivity, respectively, as measured in the late time window. Figure 8 (left) illustrates representative examples of monotonic neurons in the population, showing considerable variability in the dynamic range position and in the slope of the CRFs; one should also note that the population of monotonic cells includes some slightly supersaturating neurons, which confirms the existence of a continuum between the strictly monotonic and the selective cells (Fig. 4C). More importantly, the right panel illustrates some representative examples of selective cells with highly heterogeneous peak contrasts and bandwidths; crucially, very narrowly tuned cells exist at both low and high contrasts. The combination of these two coding strategies might be crucial for optimizing perception at the service of (different) behavioral and attentional tasks (see Discussion).

\section{Possible influence of fixed stimulus size}

In the described experiments, we used bar stimuli of fixed size $\left(2.2 \times 2.2^{\circ}\right.$; see Material and Methods $)$. This raises the possibility that the observed effects could at least partly reflect the nonoptimality of the visual drive for the recorded neurons or the compound contribution of the center and of the surround depending on RF size across the neuronal population. In particular, in early visual cortex (V1 and V2), the extent of spatial summation diminishes while surround suppression strengthens with increasing contrast and time of stimulation (Gilbert et al., 1996; Kapadia et al., 1999; Sceniak et al., 1999; Anderson et al., 2001; Cavanaugh et al., 2002; Pack et al., 2005; Henry et al., 2013). Moreover the low spatial and temporal frequencies of stimulation, as used here, have been shown to be relatively more effective in driving the suppressive surround than the excitatory portion of the RF in V1 neurons (Webb et al., 2005). Interestingly, the temporal dynamics for the emergence of contrast selectivity described here is reminiscent of the delayed intervention of sur- 

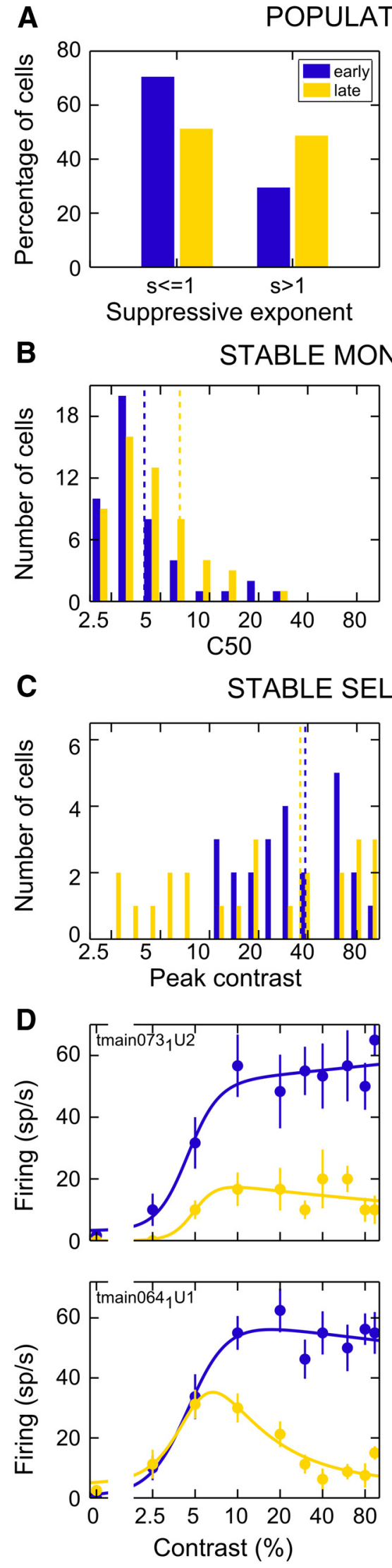

round suppression mechanisms in V1 (Bair et al., 2003; Smith et al., 2006; Henry et al., 2013). However, we carefully mapped RFs to ensure that our stimuli fell well within the classical RF, even at high contrast levels (see Materials and Methods), and we excluded cells whose RF center was located at $<2.3^{\circ}$ of eccentricity, effectively avoiding the foveal representation of V4, in which the probability of stimulating the suppressive surround might be higher because of the generally smaller RF size. In addition, systematic mapping of V4 RFs has shown that, within the range of eccentricities that we sampled, RF size varies in dimension (square root of the area) between $3.5^{\circ}$ and $9.0^{\circ}$, which is clearly above the size of our bar stimuli (Motter, 2009). Moreover, neuronal responses in V4 are maximal for bar lengths between $2^{\circ}$ and $4^{\circ}$, with suppressive effects beginning to be robust for lengths $>4^{\circ}$ ( Cheng et al., 1994, their Fig. 10 ), which is much more than the length of our stimuli. However, as a more direct test, we analyzed the incidence of selective tuning for contrast in our sample of recorded neurons as a function of RF eccentricity, which is known to covary with RF size (Desimone and Schein, 1987; Gattass et al., 1988; Motter, 2009). As shown in Figure 9, the incidence of contrast-selective cells was comparable across eccentricities, and no correlation was found between the value of the suppressive exponent $(s)$ and the eccentricity of RF centers ( $p=0.79$ ). In addition, no correlation was found between peak contrast and the eccentricity of RF centers $(p=0.25)$ in selective cells. In conclusion, the non-optimality of the visual stimulation is unlikely to have played any significant role in producing selective tuning for contrast in the present experiment.

slope (right) for the population of stably monotonic CRFs ( $s \leq$ 1.1) is shown, separately for the early and late windows (conventions as in $\boldsymbol{A}$ ). The blue and yellow vertical dotted lines represent the average $C_{50}$ and slope for the early and late windows, respectively. $C_{50}$ did not change significantly $(p=0.16$, two-tailed, paired $t$ test), whereas the slope increased significantly ( $p \ll 0.01$, two-tailed, paired $t$ test). C, Stably selective cells. The distributions of peak contrast (left) and bandwidth (right) for the population of stably selective cells $(s>1.1)$ is shown, separately for the early and late windows (conventions as in $\boldsymbol{A}$ ). Peak contrast did not change significantly ( $p=0.72$, two-tailed, paired $t$ test), whereas the bandwidth was significantly reduced in the later window $(p \ll$ 0.01 , two-tailed, paired $t$ test). $\boldsymbol{D}$, Single-cell examples. Responses of four representative $V 4$ neurons to bars of different contrast are shown. Mean firing rate [spikes per second (sp/s)] is plotted as a function of percentage Michelson contrast for the early (in blue) and late (in yellow) windows, along with the best fitted curve provided by the Peirce function (solid lines).
Figure 7. Changes in the shape of CRFs, early versus late windows. A, Entire population. In the left, the binomial distribution of the suppressive parameter $(s)$ of well fitted neurons is shown separately for the early (in blue) and late (in yellow) time windows. The right represents the percentage of suppression of each well fitted CRF. The percentage of suppression increased significantly ( $p$ $\ll 0.01$, two-tailed, paired $t$ test) from an average value of 16 to $38 \%$. $B$, Stably monotonic CRFs. The distributions of $C_{50}$ (left) and 
A

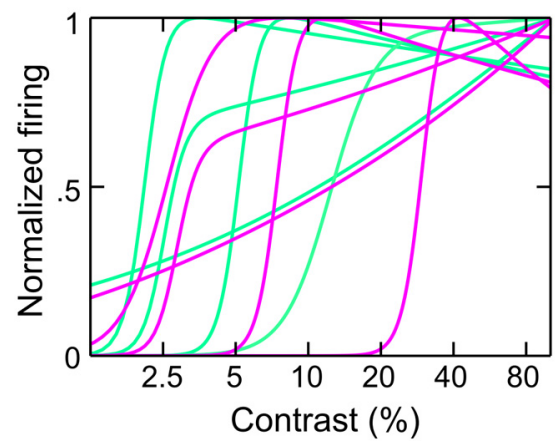

B

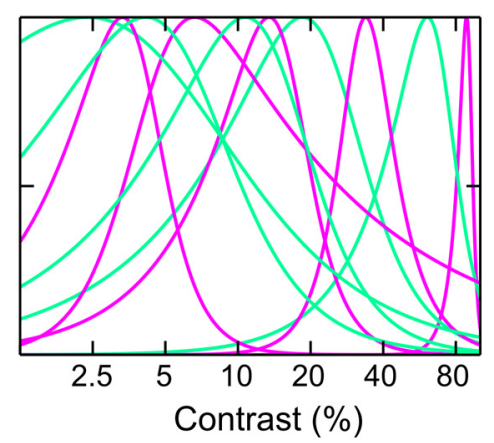

Figure 8. Filtering properties in late epochs. Normalized responses (i.e., response - baseline, divided by the maximal response) of example neurons in the late time window (100-150 ms). $\boldsymbol{A}$ shows a set of 10 neurons showing a monotonic CRF, as described by the Peirce equation ( $s \leq 1.1$ ); note that most of the functions saturate or show some degree of supersaturation. $\boldsymbol{B}$ shows a set of 10 neurons characterized by a selective CRF, as described by the Peirce equation ( $s>1.1)$; note that different neurons are selective for different contrast levels. For the sake of clarity, response functions are drawn in alternating colors along the contrast axis.

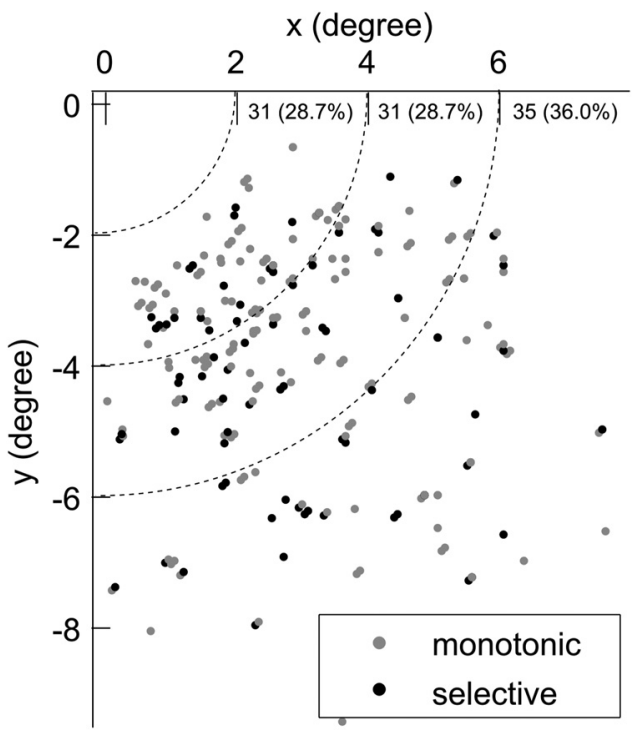

Figure 9. Contrast selectivity and eccentricity. The scatter plot depicts the RF center position within the visual field for all recorded neurons. Each cell was fitted by the Peirce function and classified as monotonic or selective according to the value of the suppressive exponent (see Results, Heterogeneous (RFs in V4; Fig. 3A). Gray and black dots correspond to neurons showing a monotonic or selective CRF, respectively. For the sake of visibility, neurons recorded at the same site are shifted by $0.05^{\circ}$. Note that contrast selectivity occurred at all levels of eccentricity, ranging from $2.53^{\circ}$ to $9.11^{\circ}$. The number and the percentage of selective cells within each sector are reported.

Nonetheless, there might be commonalities between the intervention of inhibitory mechanisms shaping bandpass tuning profiles of contrast-selective neurons in V4, as hypothesized here, and the known phenomenon of surround suppression (see Discussion).

\section{Discussion}

\section{Contrast selectivity in V4}

Here we demonstrate the existence of cells in macaque area V4 displaying bandpass tuning for contrast. These neurons are characterized by narrow filtering properties and the peaks of their CRF cover the whole range of tested contrasts, thus being similar to other types of bandpass filters that form the basis for early vision (Wilson et al., 1990). Therefore, we conjecture that, at the neurophysiological level, contrast is encoded not only as a measure of stimulus strength, or intensity (through traditional monotonic functions), but also as a basic stimulus feature, similar to other visual features, such as color or orientation. Contrast-selective tuning, as described here, potentially shares commonalities with the well known phenomenon of supersaturation, a deflection of CRFs in the high contrast range. The latter phenomenon, first recognized decades ago (Maffei and Fiorentini, 1973), has been often reported (Albrecht and Hamilton, 1982; Li and Creutzfeldt, 1984; Tyler and Apkarian, 1985; Bonds, 1991; Mancilla et al., 1998; Ledgeway et al., 2005; Peirce, 2007), although only rarely has supersaturation been examined in sufficient detail (Ledgeway et al., 2005; Peirce, 2007). The phenomenon, mainly described at early stages of the cortical visual system (mostly V1), has generally been considered as an extreme manifestation of saturation of CRFs. As a result, a systematic quantification of its relevant parameters, such as the degree of attenuation, is still lacking. Only recently, some studies raised the possibility that supersaturation might play a distinct functional role (Peirce, 2007, 2013; May and Zhaoping, 2011, 2013). Notwithstanding potential similarities, given the specific characteristics of nonmonotonic CRFs described here (including narrow peaks falling along the entire spectrum of visible contrasts), we believe that we are actually tackling a different phenomenon, more properly defined as a distinct form of selective tuning for contrast. Moreover, our results reveal that $\sim 56 \%$ of the selective cells show a suppression at high contrast $>30 \%$ in early phases of the neuronal response, whereas the proportion of cells that meet this criterion rises to $\sim 87 \%$ in later phases. Aspects of the experimental methodology (e.g., experimental paradigm, stimulus type, recording site) and data analysis in previous studies have likely contributed to largely neglecting this type of neuronal behavior. Interestingly, the proportion of contrastselective cells was similar with Gabor and bar stimuli, suggesting that the phenomenon is general and does not reflect some form of flexible coding wherein RFs are morphed according to the stimulus, as has been demonstrated for neurons in layers II and III of macaque V1 (Yeh et al., 2009).

\section{Contrast selectivity and its potential functional role}

Selective tuning for luminance contrast might support multiple functions. Our brain not only has a great capability of discriminating luminance contrast (Nachimias et al., 1973; Legge and Foley, 1980) but also of classifying it (Georgeson and Sullivan, 1975; Gottesman et al., 1981). Recent identification experiments (Chirimuuta and Tolhurst, 2005) show that humans are able to accurately categorize patterns that differ in contrast by a factor of two. This fits well with the average bandwidth of $0.3 \log$ units measured here at $150 \mathrm{~ms}$ from response onset. The proven ability of human subjects in contrast categorization could in principle rely on monotonic response functions (Chirimuuta et al., 2003), with an increasing efficiency as the steepness of the monotonic CRFs increases. However, the inclusion of bandpass tuned detectors within a population of neurons will improve both the accuracy and energy efficiency of contrast coding (May and Zhaoping, 2011) and might also reduce signal correlation across cells with 
different contrast tuning characteristics, thus yielding a potential benefit for a decoder (Averbeck et al., 2006).

Selective coding of luminance contrast is also crucial for the attentive selection of middle and low contrast stimuli. Specifically, our results are consistent with one study (Pashler et al., 2004) showing that human observers are able to efficiently select middle and low contrast stimuli among high contrast distracters. This likely reflects a form of feature-based attention mechanism directed toward any specific value of luminance contrast. In turn, a feature-based mechanism of this kind requires that specific neural populations exist that are capable of coding luminance contrast in a selective manner-exactly the capability offered by the non-monotonic neurons that we describe. More generally, this coding capability might account for other observations in which top-down guidance can override sensory-driven saliency (Ludwig and Gilchrist, 2006; Einhaüser et al., 2008) by selectively boosting the neural representation of low contrast levels.

There are two intriguing results in the psychophysical and fMRI literature that fit nicely with the present report of contrastselective neurons. Adapting to a texture of fixed contrast elements impairs the visibility of textures composed of element with straddling contrasts, indicating the presence of a contrastselective mechanism (Wolfson and Graham, 2007, 2009). The texture contrast adaptation selectivity is comparable with the narrow tuning of neurons observed here. Moreover, adapting to contrast induces a decrease of BOLD responses in early visual cortices (V1-V3) but not in V4 (Gardner et al., 2005). For this area, the BOLD response to stimuli of lower and higher contrasts relative to the adapter is paradoxically increased. This is consistent with the hypothesis that V4 is sensitive to contrast changes, as claimed by the authors, but also with the existence of neurons tuned for contrast in analogy with spatial frequency and orientation neuronal selectivity (Movshon and Blakemore, 1973; Livingstone and Hubel, 1988).

\section{Temporal dynamics of contrast selectivity and possible underlying mechanisms}

The temporal analysis of neuronal responses revealed a dynamic transformation of contrast coding, from monotonic responses to selective tuning. Early V4 responses (within $50 \mathrm{~ms}$ from stimulus onset) were predominantly monotonic and dominated by excitatory summation, along with a contribution of contrast adaptation and contrast gain mechanisms (Müller et al., 1999). Non-monotonic selectivity emerged more gradually, peaking $\sim 150 \mathrm{~ms}$ after stimulus onset. We speculate that this might correspond to a later categorization stage wherein neurons evaluate and classify contrast to support specific perceptual abilities. This transformation was evident in evolving response profiles at the population level as well as at the level of individual neurons, albeit with some variability. For most of the cells, the time taken for the development of selective tuning might reflect a time-consuming mechanism by which contrast information is recurrently encoded with gradually increasing selectivity. Recurrent processing could occur locally or could involve feedback from higher areas. However, many neurons exhibited selective tuning right after the response onset, suggesting that feedback connections from higher-level areas - which are thought to modulate rather than establish neural selectivity (Felleman and Van Essen, 1991; Bullier et al., 2001) — might not play a primary role. We hypothesize that this recurrent network architecture might correspond to a normalization mechanism almost identical to the one generating contrast saturation (Heeger, 1992; Carandini et al., 1997) or at least strongly capitalizing on its main components, with selective in- hibition for high contrasts being a decisive factor. Indeed, inhibition has been identified recently as a major determinant of spatial and temporal constrains of neuronal responses in the visual cortex of awake mice (Haider et al., 2013).

Interestingly, we also found that the time course for the emergence of contrast selectivity strikingly matches that for the refinement of orientation selectivity, supporting the idea that they might be mediated by shared or highly similar inhibitory mechanisms. In fact, it has been suggested that the shape of orientation tuning curves changes over time because of the intervention of global excitation early in the response, followed by the intervention of inhibitory circuits producing a substantial decline of the neuronal response for unpreferred orientations (Ringach et al., 1997; Shapley et al., 2003). We hypothesize that a similar biphasic involvement of excitation and inhibition might be responsible for building up selective tuning for contrast.

The intervention of inhibitory mechanisms shaping bandpass tuning profiles of contrast-selective neurons in V4, as hypothesized above, might also reflect the engagement of normalization signals similar to those deemed responsible for cross-orientation inhibition (Freeman et al., 2002; Smith et al., 2006) and to some extent contributing to orientation selectivity (Xing et al., 2011). These normalization signals are generated near the preferred spatial position of a neuron, are characterized by broad selectivity (untuned suppression; Freeman et al., 2002; Smith et al., 2006), and are mostly immune to adaptation (Freeman et al., 2002). Notably, these signals are also characterized by a very short latency, being as fast as the excitatory component (Smith et al., 2006), therefore making it rather unlikely that similar suppressive mechanisms could contribute significantly to delayed selective tuning for contrast in V4.

A potential source of inhibition might also correspond to the intervention of surround suppression mechanisms. A direct involvement of the suppressive surround of V4 neurons in generating selective tuning for contrast could be safely excluded (see Results). However, contrast tuning could be mediated by surround suppression at the level of the inputs to V4 or it may even be present at earlier processing stages. Stimuli of $\sim 2^{\circ}$ in size, as used in the present study, are large enough to directly encroach on the suppressive region of RFs, including those of V1 (Sceniak et al., 1999; Cavanaugh et al., 2002) and V2 (Solomon et al., 2004), generating a lower response at high contrast. A nonlinear pooling of the input to V4 may enhance the suppression, in turn generating the narrow selectivity. In this framework, the position of peak contrast may result from the spatial extent of the input pooling and the ratio between the strength of the center and surround responses. The temporal dynamics between excitation to the RF, which is rather short latency and transient, and the suppressive signal from the surround, which has been shown to exert a delayed and prolonged effect on neuronal firing in V1 (Bair et al., 2003; Webb et al., 2005; Smith et al., 2006; Henry et al., 2013), is also consistent with the delayed emergence of full blown contrast selectivity after visual stimulation, as described here. However, it is important to note that any described delay of suppression in V1 occurs within a relatively short timescale (within 10-60 ms after the onset of the excitatory component; Bair et al., 2003; Smith et al., 2006; Henry et al., 2013), whereas the maximal expression of contrast selectivity that we describe here in $\mathrm{V} 4 \mathrm{oc}-$ curs considerably later in time, $\sim 120 \mathrm{~ms}$ after response onset.

It has been suggested that surround suppression is likely to serve a number of purposes, including redundancy reduction (Atick, 1992), noise rejection (Chen et al., 2006), figure-ground segmentation (Allman et al., 1985), and feature detection (Hubel 
and Wiesel, 1965), but also more generic functions of cortical networks, such as normalization and gain control (Tsui and Pack, 2011). It may well also serve the generation of contrast tuning, of the type observed here. However, whatever the local circuits mediating the emergence of the contrast tuning, the evidence of a narrow tuning selectivity and of a wide spread of preferred contrasts point to a functional role of the phenomenon possibly related to contrast categorization and the generation of filters that can be selected by attention.

We speculate that contrast selectivity might be a slowly emerging property along the visual system, with $\mathrm{V} 4$ perhaps playing a key role, as also shown by the inability to select relatively less prominent stimuli-including low contrast stimuli-after V4 lesions (Schiller, 1993; De Weerd et al., 1999). In this regard, our results are in line with the recently proposed notion that the unifying function of V4 circuitry is to enable "selective extraction" by facilitating figure-ground segmentation of the visual scene and attentional filtering (Roe et al., 2012). According to this view, contrast selectivity would result from the dynamical configuration of a feature extraction network, supporting specific functions such as contrast identification and the attentive selection of low contrast stimuli. The current demonstration that coding for contrast in macaque area V4 is highly heterogeneous and is critically sculpted by a time-consuming mechanism now calls for a reexamination of the interplay between spatial selective attention and contrast in this brain area.

\section{References}

Albrecht DG (1995) Visual cortex neurons in monkey and cat: effect of contrast on the spatial and temporal phase transfer functions. Vis Neurosci 12:1191-1210. CrossRef Medline

Albrecht DG, Hamilton DB (1982) Striate cortex of monkey and cat: contrast response function. J Neurophysiol 48:217-237. Medline

Albrecht DG, Geisler WS, Frazor RA, Crane AM (2002) Visual cortex neurons of monkeys and cats: temporal dynamics of the contrast response function. J Neurophysiol 88:888-913. Medline

Allman J, Miezin F, McGuinness E (1985) Direction- and velocity-specific responses from beyond the classical receptive field in the middle temporal visual area (MT). Perception 14:105-126. CrossRef Medline

Anderson JS, Lampl I, Gillespie DC, Ferster D (2001) Membrane potential and conductance changes underlying length tuning of cells in cat primary visual cortex. J Neurosci 21:2104-2112. Medline

Atick JJ (1992) Could information theory provide an ecological theory of sensory processing? Network Comp Neural 3:213-251.

Averbeck BB, Latham PE, Pouget A (2006) Neural correlations, population coding and computation. Nat Rev Neurosci 7:358-366. CrossRef Medline

Bair W, Cavanaugh JR, Movshon JA (2003) Time course and time-distance relationships for surround suppression in macaque V1 neurons. J Neurosci 23:7690-7701. Medline

Barlow HB, Blakemore C, Pettigrew JD (1967) The neural mechanism of binocular depth discrimination. J Physiol 193:327-342. Medline

Bonds AB (1991) Temporal dynamics of contrast gain single cells of the cat striate cortex. Vis Neurosci 6:239-255. CrossRef Medline

Bullier J, Hupé JM, James AC, Girard P (2001) The role of feedback connections in shaping the responses of visual cortical neurons. Prog Brain Res 134:193-204. CrossRef Medline

Carandini M, Heeger DJ, Movshon JA (1997) Linearity and normalization in simple cells of the macaque primary visual cortex. J Neurosci 17:86218644. Medline

Cavanaugh JR, Bair W, Movshon JA (2002) Nature and interaction of signals from the receptive field center and surround in macaque V1 neurons. J Neurophysiol 88:2530-2546. CrossRef Medline

Chen Y, Geisler WS, Seidemann E (2006) Optimal decoding of correlated neural population responses in the primate visual cortex. Nat Neurosci 9:1412-1420. CrossRef Medline

Cheng K, Hasegawa T, Saleem KS, Tanaka K (1994) Comparison of neuronal selectivity for stimulus speed, length, and contrast in the prestriate visual cortical areas V4 and MT of the macaque monkey. J Neurophysiol 71:2269-2280. Medline

Chirimuuta M, Tolhurst DJ (2005) Accuracy of identification of grating contrast by human observers: Bayesian models of $\mathrm{V} 1$ contrast processing show correspondence between discrimination and identification performance. Vision Res 45:2960-2971. CrossRef Medline

Chirimuuta M, Clatworthy PL, Tolhurst DJ (2003) Coding of the contrasts in natural images by visual cortex (V1) neurons: a Bayesian approach. J Opt Soc Am A Opt Image Sci Vis 20:1253-1260. CrossRef Medline

Desimone R, Schein SJ (1987) Visual properties of neurons in area V4 of the macaque: sensitivity to stimulus form. J Neurophysiol 57:835-868. Medline

De Weerd P, Peralta MR 3rd, Desimone R, Ungerleider LG (1999) Loss of attentional stimulus selection after extrastriate cortical lesions in macaques. Nat Neurosci 2:753-758. CrossRef Medline

Einhäuser W, Rutishauser U, Koch C (2008) Task-demands can immediately reverse the effects of sensory-driven saliency in complex visual stimuli. J Vis 8:2 1-19. CrossRef Medline

Enroth-Cugell C, Shapley RM (1973) Adaptation and dynamics of cat retinal ganglion cells. J Physiol 233:271-309. Medline

Felleman DJ, Van Essen DC (1991) Distributed hierarchical processing in the primate cerebral cortex. Cereb Cortex 1:1-47. CrossRef Medline

Freeman TC, Durand S, Kiper DC, Carandini M (2002) Suppression without inhibition in visual cortex. Neuron 35:759-771. CrossRef Medline

Gardner JL, Sun P, Waggoner RA, Ueno K, Tanaka K, Cheng K (2005) Contrast adaptation and representation in human early visual cortex. Neuron 47:607-620. CrossRef Medline

Gattass R, Sousa AP, Gross CG (1988) Visuotopic organization and extent of V3 and V4 of the macaque. J Neurosci 8:1831-1845. Medline

Gawne TJ, Kjaer TW, Richmond BJ (1996) Latency: another potential code for feature binding in striate cortex. J Neurophysiol 76:1356-1360. Medline

Georgeson MA, Sullivan GD (1975) Contrast constancy: deblurring in human vision by spatial frequency channels. J Physiol 252:627-656. Medline

Gilbert CD, Das A, Ito M, Kapadia M, Westheimer G (1996) Spatial integration and cortical dynamics. Proc Natl Acad Sci U S A 93:615-622. CrossRef Medline

Gottesman J, Rubin GS, Legge GE (1981) A power law for perceived contrast in human vision. Vision Res 21:791-799. CrossRef Medline

Haider B, Häusser M, Carandini M (2013) Inhibition dominates sensory responses in the awake cortex. Nature 493:97-100. CrossRef Medline

Heeger DJ (1992) Normalization of cell responses in cat striate cortex. Vis Neurosci 9:181-197. CrossRef Medline

Henry CA, Joshi S, Xing D, Shapley RM, Hawken MJ (2013) Functional characterization of the extraclassical receptive field in macaque V1: contrast, orientation, and temporal dynamics. J Neurosci 33:6230-6242. CrossRef Medline

Hu M, Wang Y, Wang Y (2011) Rapid dynamics of contrast responses in the cat primary visual cortex. PLoS One 6:e25410. CrossRef Medline

Hubel DH, Wiesel TN (1965) Receptive fields and functional architecture in two nonstriate visual areas (18 and 19) of the cat. J Neurophysiol 28:229 289. Medline

Kapadia MK, Westheimer G, Gilbert CD (1999) Dynamics of spatial summation in primary visual cortex of alert monkeys. Proc Natl Acad Sci U S A 96:12073-12078. CrossRef Medline

Ledgeway T, Zhan C, Johnson AP, Song Y, Baker CL Jr (2005) The direction-selective contrast response of area 18 neurons is different for first- and second-order motion. Vis Neurosci 22:87-99. CrossRef Medline

Lee J, Maunsell JH (2009) A normalization model of attentional modulation of single unit responses. PLoS One 4:e4651. CrossRef Medline

Lee J, Williford T, Maunsell JH (2007) Spatial attention and the latency of neuronal responses in macaque area V4. J Neurosci 27:9632-9637. CrossRef Medline

Legge GE, Foley JM (1980) Contrast masking in human vision. J Opt Soc Am 70:1458-1471. Medline

Li CY, Creutzfeldt O (1984) The representation of contrast and other stimulus parameters by single neurons in area 17 of the cat. Pflugers Arch 401:304-314. CrossRef Medline

Lisberger SG, Movshon JA (1999) Visual motion analysis for pursuit eye movements in area MT of macaque monkeys. J Neurosci 19:2224-2246. Medline 
Livingstone M, Hubel D (1988) Segregation of form, color, movement, and depth: anatomy, physiology, and perception. Science 240:740-749. CrossRef Medline

Ludwig CJH, Gilchrist ID (2006) The relative contributions of luminance contrast and task demands on saccade target selection. Vision Res 46: 2743-2748. CrossRef Medline

Maffei L, Fiorentini A (1973) The visual cortex as a spatial frequency analyser. Vision Res 13:1255-1267. CrossRef Medline

Maffei L, Fiorentini A, Bisti S (1973) Neural correlate to perceptual adaptation to gratings. Science 182:1036-1038. CrossRef Medline

Mancilla JG, Fowler M, Ulinski PS (1998) Responses of regular spiking and fast spiking cells in turtle visual cortex to light flashes. Vis Neurosci 15: 979-993. Medline

May KA, Zhaoping L (2011) Exploring the roles of saturating and supersaturating contrast-response functions in conjunction detection and contrast coding. J Vis 11:11. CrossRef Medline

May KA, Zhaoping L (2013) The potential roles of saturating and supersaturating contrast-response functions in conjunction detection: reply to Peirce. J Vis 13:22. CrossRef Medline

McAdams CJ, Maunsell JH (1999) Effects of attention on the reliability of individual neurons in monkey visual cortex. Neuron 23:765-773. CrossRef Medline

Mirabella G, Bertini G, Samengo I, Kilavik BE, Frilli D, Della Libera C, Chelazzi L (2007) Neurons in area V4 of the macaque translate attended visual features into behaviorally relevant categories. Neuron 54:303-318. CrossRef Medline

Motter BC (2009) Central V4 receptive fields are scaled by the V1 cortical magnification and correspond to a constant-sized sampling of the V1 surface. J Neurosci 29:5749-5757. CrossRef Medline

Motulsky H, Christopoulos A (2004) Fitting models to biological data using linear and nonlinear regression: a practical guide to curve fitting. New York: Oxford UP.

Movshon JA, Blakemore C (1973) Orientation specificity and spatial selectivity in human vision. Perception 2:53-60. CrossRef Medline

Movshon JA, Lennie P (1979) Pattern-selective adaptation in visual cortical neurones. Nature 278:850-852. CrossRef Medline

Müller JR, Metha AB, Krauskopf J, Lennie P (1999) Rapid adaptation in visual cortex to the structure of images. Science 285:1405-1408. CrossRef Medline

Nachimias J, Sansbury R, Vassilev A, Weber A (1973) Adaptation to squarewave gratings: in search of the elusive third harmonic. Vision Res 13: 1335-1342. CrossRef Medline

Pack CC, Hunter JN, Born RT (2005) Contrast dependence of suppressive influences in cortical area MT of alert macaque. J Neurophysiol 93:18091815. Medline

Pashler H, Dobkins K, Huang L (2004) Is contrast just another feature for visual selective attention? Vis Res 44:1403-1410. Medline

Peirce JW (2007) The potential importance of saturating and supersaturating contrast response functions in visual cortex. J Vis 7:13. CrossRef Medline

Peirce JW (2013) Nonlinear response functions can still be used to build conjunction detectors: reply to May and Zhaoping. J Vis 13:23. CrossRef Medline

Reynolds JH, Heeger DJ (2009) The normalization model of attention. Neuron 61:168-185. CrossRef Medline
Reynolds JH, Pasternak T, Desimone R (2000) Attention increases sensitivity of V4 neurons. Neuron 26:703-714. CrossRef Medline

Ringach DL, Hawken MJ, Shapley R (1997) Dynamics of orientation tuning in macaque primary visual cortex. Nature 387:281-284. CrossRef Medline

Roe AW, Chelazzi L, Connor CE, Conway BR, Fujita I, Gallant JL, Lu H, Vanduffel W (2012) Toward a unified theory of visual area V4. Neuron 74:12-29. CrossRef Medline

Sceniak MP, Ringach DL, Hawken MJ, Shapley R (1999) Contrast's effect on spatial summation by macaque V1 neurons. Nat Neurosci 2:733-739. CrossRef Medline

Schiller PH (1993) The effects of V4 and middle temporal (MT) area lesions on visual performance in the rhesus monkey. Vis Neurosci 10:717-746. Medline

Sclar G, Lennie P, DePriest DD (1989) Contrast adaptation in striate cortex of macaque. Vision Res 29:747-755. CrossRef Medline

Shapley R, Hawken M, Ringach DL (2003) Dynamics of orientation selectivity in the primary visual cortex and the importance of cortical inhibition. Neuron 38:689-699. CrossRef Medline

Sharpee TO, Sugihara H, Kurgansky AV, Rebrik SP, Stryker MP, Miller KD (2006) Adaptive filtering enhances information transmission in visual cortex. Nature 439:936-942. CrossRef Medline

Smith MA, Bair W, Movshon JA (2006) Dynamics of suppression in macaque primary visual cortex. J Neurosci 26:4826-4834. CrossRef Medline

Solomon SG, Peirce JW, Lennie P (2004) The impact of suppressive surrounds on chromatic properties of cortical neurons. J Neurosci 24:148 160. CrossRef Medline

Thiele A, Pooresmaeili A, Delicato LS, Herrero JL, Roelfsema PR (2009) Additive effects of attention and stimulus contrast in primary visual cortex. Cereb Cortex 19:2970-2981. CrossRef Medline

Tsui JM, Pack CC (2011) Contrast sensitivity of MT receptive field centers and surrounds. J Neurophysiol 106:1888-1900. CrossRef Medline

Tyler CW, Apkarian PA (1985) Effects of contrast, orientation and binocularity in the pattern evoked potential. Vision Res 25:755-766. CrossRef Medline

Walker GA, Ohzawa I, Freeman RD (2000) Suppression outside the classical cortical receptive field. Vis Neurosci 17:369-379. Medline

Webb BS, Dhruv NT, Solomon SG, Tailby C, Lennie P (2005) Early and late mechanisms of surround suppression in striate cortex of macaque. J Neurosci 25:11666-11675. CrossRef Medline

Williford T, Maunsell JH (2006) Effects of spatial attention on contrast response functions in macaque area V4. J Neurophysiol 96:40-54. CrossRef Medline

Wilson HR, Levi D, Maffei L, Rovamo J, DeValois R (1990) Visual perception: the neurophysiological foundations (Spillmann L, Werner JS, eds), pp 231-272. San Diego: Academic.

Wolfson SS, Graham N (2007) An unusual kind of contrast adaptation: shifting a contrast comparison level. J Vis 7:12. CrossRef Medline

Wolfson SS, Graham N (2009) Two contrast adaptation processes: contrast normalization and shifting, rectifying contrast comparison. J Vis 9:30 1-23. CrossRef Medline

Xing D, Ringach DL, Hawken MJ, Shapley RM (2011) Untuned suppression makes a major contribution to the enhancement of orientation selectivity in macaque V1. J Neurosci 31:15972-15982. CrossRef Medline

Yeh CI, Xing D, Shapley RM (2009) "Black" responses dominate macaque primary visual cortex V1. J Neurosci 29:11753-11760. CrossRef Medline 